\title{
Competitive Equilibria of Economies with a Continuum of Consumers and Aggregate Shocks ${ }^{1}$
}

\author{
JIANJUN MIAO²
}

December 2004

\begin{abstract}
${ }^{1}$ I thank Larry Epstein for constant support and guidance. I am deeply indebted for his insightful and detailed comments. I have benefited also from helpful discussions with Jim Bergin, Dan Bernhardt, Mark Huggett, Per Krusell, Felix Kubler, Kevin Reffett, Manuel Santos, and seminar participants at ASU, Carnegie Mellon University, the 2002 Midwest Economic Theory Conference, the 2002 North American Summer Meeting of the Econometric Society, and the 2002 NBER Conference on General Equilibrium Theory. I am especially grateful to an anonymous referee for thoughtful comments and suggestions.

${ }^{2}$ Department of Economics, Boston University, 270 Bay State Road, Boston, MA 02215. Email: miaoj@bu.edu. Homepage: http://people.bu.edu/miaoj. Tel: 617-353-6675. Fax: 617-353-4449.
\end{abstract}




\begin{abstract}
This paper studies competitive equilibria of a production economy with aggregate productivity shocks. There is a continuum of consumers who face borrowing constraints and individual labor endowment shocks. The dynamic economy is described in terms of sequences of aggregate distributions. The existence of sequential competitive equilibria is proven and a recursive characterization is established. In particular, it is shown that for any sequential competitive equilibrium, there exists a payoff equivalent sequential competitive equilibrium that is generated by a suitably defined recursive equilibrium with state variables including continuation value.
\end{abstract}

Key words: competitive equilibrium, recursive equilibrium, aggregate distribution, heterogeneity, incomplete markets

JEL Classification Numbers: D50, D52, E20 


\section{INTRODUCTION}

It has been documented by a number of empirical studies that the standard representative agent (or complete markets) model fails to explain many phenomena observed in the data. This leads to interest in models with heterogeneity and incomplete markets. ${ }^{1}$ One class of such models, called the Bewley-style model, has drawn special attention. A typical environment of this model is described in Krusell and Smith [24]. It features a continuum of consumers making consumption and savings decisions subject to borrowing constraints and idiosyncratic labor endowment shocks. There is only one asset (capital) serving as a buffer against individual shocks. Finally, a representative firm makes production decisions with constant-returns-to-scale technology, subject to aggregate productivity shocks. ${ }^{2}$

This paper addresses two central open questions. The first is whether there exist sequential competitive equilibria for this type of model. The second is whether there exists a recursive characterization of sequential competitive equilibria. Krusell and Smith [24] and a number of later studies pose directly a recursive equilibrium formulation (henceforth, KS-recursive equilibrium) and then proceed with numerical solutions without studying its existence or the relation to sequential competitive equilibria. By contrast, I start with the analysis of sequential competitive equilibria and then move on to recursive characterizations.

The key insight of this paper is to reformulate the Bewley-style model along the lines of Hildenbrand [18] and Hart et al. [15]. Specifically, I describe the dynamic economy using sequences of aggregate distributions over consumers' characteristics (individual asset holdings and the realization of endowment shocks) across the population. These sequences of aggregate distributions contain the relevant information for equilibrium analysis and they are the principal objects of study. In particular, given exogenous shocks, aggregate distributions fully determine prices and aggregate quantities such as aggregate capital. It turns out that this reformulation is the key to answering the preceding two questions.

To study the existence of sequential competitive equilibria, I begin with a detailed analysis of a typical individual's decision problem. After aggregating individual optimal behavior and deriving the law of motion for aggregate distributions, I establish the existence of a sequential competitive equilibrium by applying the Brouwer-Schauder-Tychonoff Fixed-Point Theorem to a compact space of sequences of aggregate distributions (Theorem 1). This result is established

\footnotetext{
${ }^{1}$ See the survey by Heaton and Lucas [16] and the textbook by Ljunqvist and Sargent [28].

${ }^{2}$ See $[8,9,2,19,31]$ for Bewley-style models without aggregate shocks.
} 
under standard assumptions on preferences and technology and for fairly general individual and aggregate shock processes. For example, these are assumed to satisfy the Feller property, but they need not be stationary or Markovian. However, for technical reasons, I assume that the state space for aggregate shocks is countable.

After imposing the additional assumption that individual and aggregate shocks are timehomogenous Markov processes, I turn to recursive characterizations of sequential competitive equilibria. I define a notion of recursive equilibrium with the state variables consisting of individual asset holdings, the realization of individual shocks, the realization of aggregate shocks, the aggregate distribution, and payoffs (expected discounted utilities). Including the first three as state variables is standard. It is also natural to include the aggregate distribution as a state variable because with incomplete markets and heterogeneous consumers, equilibrium prices generally depend on the distribution of assets across consumers.

Including payoffs (or continuation value) as a state variable to make certain decision problems recursive is a technique widely adopted in the literature on sequential games $[11,5,7]$ and on dynamic contracts $[32,36,1]$. Here this state variable serves as a device for selecting continuation equilibria when the economy unfolds over time.

Theorem 2 demonstrates that given an initial state, the so defined recursive equilibrium generates a sequential competitive equilibrium. Theorem 3 demonstrates that a recursive equilibrium exists. Moreover, for any sequential competitive equilibrium, there is a payoff equivalent sequential competitive equilibrium that is generated by a recursive equilibrium with the state space including payoffs.

A natural but open question is whether there is a recursive equilibrium with a smaller state space, for example, the KS-recursive equilibrium that excludes expected payoffs as a state variable. For a related finitely many agents economy, Kubler and Schmedders [25, Theorem 2] establish the existence of such a recursive equilibrium under the strong condition that the competitive equilibrium is globally unique for all possible initial values. Although one can state a similar result for the economies studied here, this strong condition cannot be checked from primitives.

The above analysis must surmount two technical difficulties. First, there is a difficulty associated with the presence of aggregate shocks. When they are present, aggregate distributions are generally random measures that may be correlated with individual shocks. As pointed out by Bergin and Bernhardt [6], this creates not only difficulties of tractability but also concep- 
tual problems associated with the meaning of perfect competition. Thus, I follow Bergin and Bernhardt [6] and assume the conditional no aggregate uncertainty condition. This requires that, conditional on the history of aggregate shocks, the aggregate distribution at each date be a constant measure. Second, there are subtle technical problems, pointed out by Judd [20], associated with an environment that has a continuum of agents, e.g., measurability and the law of large numbers. This paper deals with these problems in a manner similar to Feldman and Gilles [13] and Karatzas et al. [21]. ${ }^{3}$

I now review the related literature. There is a growing literature on numerical analysis of Bewley-style models with aggregate shocks $[23,24,14,34]$. None of these considers the theoretical issues studied here. My paper is also related to [11, 4]. There is also an extensive literature on sequential competitive equilibria for pure-exchange incomplete markets economies (e.g., $[29,27,17,30])$. All these papers consider a finite number of heterogeneous consumers. The traditional method of proving existence of sequential competitive equilibria is to take limits of equilibria in truncated economies in which trade stops at some finite date. This requires two conditions: First, the existence of sequential competitive equilibria for finite-horizon economies must be established. Second, the resulting sequence of equilibria for finite-horizon economies must converge to a limit under some suitable topology and this limit is the equilibrium of the original infinite-horizon economy. By contrast, this paper follows a direct proof strategy. Exploiting the special feature of the continuum agents environment, I reformulate the economy in terms of sequences of aggregate distributions. The space of these sequences endowed with some topology is compact, and hence a topological fixed point theorem can be applied if a suitably defined map is continuous. A sequential competitive equilibrium is then delivered by a fixed point of this map. This proof method is much simpler than the traditional one.

In order to ensure compactness, I impose exogenously fixed borrowing constraints, instead of "non-binding" endogenous borrowing constraints (that is, borrowing constraints that rule out Ponzi schemes but nothing else). This assumption is also adopted in Duffie et al. [11] and the applied macroeconomics literature. My proof method does not apply to the case of non-binding endogenous borrowing constraints. Moreover, the existence of continuous recursive equilibria may not be guaranteed if the exogenous borrowing constraints never bind. This is because under this condition Krebs [22] proves a nonexistence result for pure-exchange economies with finitely many agents. Whether or not a similar nonexistence result holds in the economy studied here is an open question.

\footnotetext{
${ }^{3}$ Also see an alternative approach proposed by Sun [35].
} 
The recursive characterization in this paper is different from Duffie et al. [11] who study finitely many agents economies. ${ }^{4}$ The key idea of Duffie et al. [11] is to construct an expectations correspondence, which specifies, for each possible current state, the transitions that are consistent with feasibility and satisfies short-run equilibrium conditions. Typically, the expectations correspondence is constructed using the first-order conditions for all agents. This procedure seems invalid for the continuum agents economies since there is a continuum of first-order conditions. Moreover, my result does not require differentiability assumptions on utility functions, so first-order conditions are not available. My recursive characterization is achieved by first defining an equilibrium correspondence and a related correspondence, and then taking a measurable selection. An advantage of my recursive characterization is that the state space is smaller than that in Duffie et al. [11] since the latter generally includes all endogenous variables, exogenous shocks, and signal variables as state variables.

The remainder of the paper proceeds as follows. Section 2 sets up the model. Section 3 analyzes the existence of a sequential competitive equilibrium. Section 4 studies recursive characterizations of sequential competitive equilibria. Section 5 concludes. Proofs are relegated to an appendix.

\section{THE MODEL}

Consider an economy with a large number of infinitely-lived consumers subject to individual endowment shocks and a single firm subject to aggregate productivity shocks. This economy is similar to that studied by Krusell and Smith [24]. Time is discrete and denoted by $t=0,1,2, \ldots$. Uncertainty is represented by a probability space $\left(\Omega \times \mathbb{Z}^{\infty}, \mathcal{F}, P\right)$ on which all stochastic processes are defined. The state space $\Omega$ captures individual shocks, while the state space $\mathbb{Z}^{\infty}$ captures aggregate shocks. Let $\mathbb{Z}^{0}=\mathbb{Z}, \mathbb{Z}^{t+1}=\mathbb{Z}^{0} \times \mathbb{Z}^{t}, z^{0}=z_{0}$, and denote by $z^{t}=\left(z_{0}, z_{1}, \ldots, z_{t}\right) \in \mathbb{Z}^{t}$ an aggregate shock history at time $t$. Finally, let $z^{\infty}=\left(z_{0}, z_{1}, z_{2}, \ldots\right) \in \mathbb{Z}^{\infty}$ be the complete history and $z^{0}=z_{0} \in \mathbb{Z}^{0}$ be a deterministic constant.

Notation. For any Euclidean subspace $\mathbb{D}$, denote by $\mathbb{C}(\mathbb{D})$ the space of bounded and continuous functions on $\mathbb{D}$ endowed with the sup-norm, by $\mathcal{B}(\mathbb{D})$ the Borel $\sigma$-algebra of $\mathbb{D}$, and by $\mathcal{P}(\mathbb{D})$ the space of probability measures on $\mathcal{B}(\mathbb{D})$ endowed with the weak convergence topology. For any

\footnotetext{
${ }^{4}$ One important difference is that Duffie et al [11] study recursive equilibria that admit ergodic distributions, while I do not consider existence of ergodic distributions. The idea of Duffie et al. [11] is pursued further by Kubler and Schmedders [25, 26] for exchange economies.
} 
Euclidean sets $\mathbb{D}$ and $\mathbb{E}, \mathcal{B}(\mathbb{D}) \otimes \mathcal{B}(\mathbb{E})$ denotes the product $\sigma$-algebra.

\subsection{Consumers}

Consumers are distributed on the interval $I=[0,1]$ according to the Lebesgue measure $\phi$. Consumers are ex ante identical in that they have the same preferences and their endowment shock processes are drawn from the same distribution. However, consumers are ex post heterogeneous in the sense that they experience idiosyncratic endowment shocks. ${ }^{5}$

Information structure and endowments. Consumer $i \in I$ is endowed with one unit of labor at each date $t$ and a deterministic asset level $a_{0}^{i} \in \mathbb{R}_{++}$at the beginning of time 0 . Labor endowment is subject to random shocks represented by a stochastic process $\left(s_{t}^{i}\right)_{t \geq 0}$ valued in $\mathbb{S} \subset \mathbb{R}_{+}$, where $s_{0}^{i}$ is a deterministic constant. Let $\mathbb{S}^{0}=\mathbb{S}, \mathbb{S}^{t+1}=\mathbb{S}^{0} \times \mathbb{S}^{t}, s^{0 i}=s_{0}^{i}$, and denote by $s^{t i}=\left(s_{0}^{i}, s_{1}^{i}, \ldots, s_{t}^{i}\right) \in \mathbb{S}^{t}$ an individual shock history. Let the initial (probability) distribution of asset holdings and endowment shocks be given by

$$
\lambda_{0}(A \times S)=\phi\left(i \in I:\left(a_{0}^{i}, s_{0}^{i}\right) \in A \times S\right), A \times S \in \mathcal{B}\left(\mathbb{R}_{++}\right) \times \mathcal{B}(\mathbb{S}) .
$$

At the beginning of date $t$, consumer $i$ observes his labor endowment shock $s_{t}^{i}$ and the aggregate productivity shock $z_{t}$. His information is represented by a $\sigma$-algebra $\mathcal{F}_{t}^{i}$ generated by past and current shocks $\left\{s_{n}^{i}, z_{n}\right\}_{n=0}^{t} \cdot{ }^{6}$ The following assumptions on the shock processes are maintained.

Assumption $1 \mathbb{Z} \subset[\underline{z}, \bar{z}] \subset \mathbb{R}_{++}$is a bounded and countable set endowed with the discrete topology; $\mathbb{S} \subset \mathbb{R}_{+}$is compact.

Assumption 2 For $\phi$-a.e. $i$,

(a) given the history $\left(s^{i t}, z^{t}\right)=\left(s^{t}, z^{t}\right),\left(s_{t+1}^{i}, z_{t+1}\right)$ is drawn from the distribution $Q_{t+1}\left(\cdot, s^{t}, z^{t}\right)$;

(b) $Q_{t+1}(S \times Z, \cdot)$ is measurable for all $S \times Z \in \mathcal{B}(\mathbb{S}) \times \mathcal{B}(\mathbb{Z})$;

(c) $Q_{t+1}$ has the Feller property: $\int h\left(s^{\prime}, z^{\prime}\right) Q_{t+1}\left(d s^{\prime}, d z^{\prime}, \cdot\right)$ is a continuous function on $\mathbb{S}^{t} \times \mathbb{Z}^{t}$ for any real-valued, bounded, and continuous function $h$ on $\mathbb{S} \times \mathbb{Z}$.

\footnotetext{
${ }^{5}$ The extension to the case of ex ante heterogeneous consumers is in the working paper version of the paper, which is available upon request.

${ }^{6}$ Alternatively, one can consider the case where each consumer observes the aggregate shocks after he makes choices so that $\mathcal{F}_{t}^{i}$ is generated by $\left\{s_{n}^{i}, z_{n-1}\right\}_{n=0}^{t}, z_{-1}$ is null.
} 
Remark 2.1 It merits emphasis that the state space of aggregate shocks is assumed to be countable, which avoids measurability problems that may arise in dynamic programming. See [7] for the treatment when this space is uncountable.

Consumption Space. There is a single good. A consumption plan $c^{i} \equiv\left(c_{t}^{i}\right)_{t=0}^{\infty}$ for consumer $i$ is a nonnegative real-valued process such that $c_{t}^{i}$ is $\mathcal{F}_{t}^{i}$-measurable. ${ }^{7}$ Denote by $\mathcal{C}^{i}$ the set of all consumption plans for consumer $i$.

Budget and borrowing constraints. An asset accumulation plan $\left(a_{t+1}^{i}\right)_{t \geq 0}$ for consumer $i$ is a real-valued process such that $a_{t+1}^{i}$ is $\mathcal{F}_{t}^{i}$-measurable.

In each period $t$, consumer $i$ consumes $c_{t}^{i}$ and accumulates assets $a_{t+1}^{i}$ subject to the familiar budget constraint:

$$
c_{t}^{i}+a_{t+1}^{i}=\left(1+r_{t}\right) a_{t}^{i}+w_{t} s_{t}^{i}, a_{0}^{i} \text { given, }
$$

where $r_{t}$ is the rental rate and $w_{t}$ is the wage rate. For simplicity, assume that all consumers cannot borrow so that:

$$
a_{t+1}^{i} \geq 0 \text { for all } i \in I \text {. }
$$

Finally, let $\mathbb{A}=[0, \infty)$, and denote by $\mathcal{A}^{i}$ the set of all asset accumulation plans of consumer $i$ that satisfy the budget constraint (1) and the borrowing constraint (2). A consumption plan $c \in \mathcal{C}^{i}$ corresponding to an asset accumulation plan $a \in \mathcal{A}^{i}$ is called (budget) feasible.

Preferences. Consumer $i$ 's preferences are represented by an expected utility function defined on $\mathcal{C}^{i}:$

$$
U\left(c^{i}\right)=E\left[\sum_{t=0}^{\infty} \beta^{t} u\left(c_{t}^{i}\right)\right],\left(c_{t}^{i}\right) \in \mathcal{C}^{i},
$$

where $\beta \in(0,1)$ is the discount factor and $u: \mathbb{R}_{+} \rightarrow \mathbb{R}$ is the felicity function satisfying:

Assumption 3 The function $u$ is bounded, continuous, and strictly concave.

Decision problem. Consumer $i$ 's problem is given by:

$$
\sup _{\left(c_{t}^{i}, a_{t+1}^{i}\right)_{t \geq 0} \in \mathcal{C}^{i} \times \mathcal{A}^{i}} U\left(c^{i}\right) .
$$

\footnotetext{
${ }^{7}$ Because of this measurability, I may write the value of $c_{t}^{i}$ at state $\left(\omega, z^{\infty}\right)$ for consumer $i \operatorname{simply}$ as $c_{t}^{i}\left(\omega, z^{t}\right)$. Similar notation applies to other adapted processes.
} 
The plans $\left(c_{t}^{i}\right)_{t \geq 0}$ and $\left(a_{t+1}^{i}\right)_{t \geq 0}$ are optimal if the above supremum is achieved by $\left(c_{t}^{i}, a_{t+1}^{i}\right)_{t \geq 0} \in$ $\mathcal{C}^{i} \times \mathcal{A}^{i}$

Allocation. An allocation $\left(\left(c_{t}^{i}, a_{t+1}^{i}\right)_{t \geq 0}\right)_{i \in I}$ is a collection of consumption and asset accumulation plans $\left(c_{t}^{i}, a_{t+1}^{i}\right)_{t \geq 0}, i \in I$. An allocation $\left(\left(c_{t}^{i}, a_{t+1}^{i}\right)_{t \geq 0}\right)_{i \in I}$ is admissible if both $c_{t}^{i}=c_{t}\left(i, \omega, z^{t}\right)$ and $a_{t+1}^{i}=a_{t+1}\left(i, \omega, z^{t}\right)$ are $\mathcal{B}(I) \otimes \mathcal{F}_{t}$-measurable where $\mathcal{F}_{t}$ is the smallest $\sigma$-algebra containing $\mathcal{F}_{t}^{i}$ for all $i \in I, \mathcal{F}_{t}=\vee_{i \in I} \mathcal{F}_{t}^{i}, t \geq 0$. This measurability requirement ensures certain integrals are well defined (see [10] for discussion of the difficulties that arise if it is violated). Since both $c_{t}^{i}$ and $a_{t+1}^{i}$ are $\mathcal{F}_{t}^{i}$-measurable for all fixed $i \in I$, they are also $\mathcal{F}_{t}$-measurable. Thus, the essential content of admissibility is that $c_{t}^{i}$ and $a_{t+1}^{i}$ must be $\mathcal{B}(I)$-measurable for each fixed $\left(\omega, z^{t}\right) \in \Omega \times \mathbb{Z}^{t}$. To ensure that admissible allocations exist, I assume: ${ }^{8}$

Assumption 4 For each $t, s_{t}: I \times \Omega \times \mathbb{Z}^{\infty} \rightarrow \mathbb{S}$ is $\mathcal{B}(I) \otimes \mathcal{F}_{t}$-measurable.

\subsection{The Firm}

There is a single firm renting capital at (net) rate $r_{t}$ and hiring labor at wage $w_{t}$ at date $t$. It produces output $Y_{t}$ with the constant-returns-to-scale technology $F: \mathbb{R}_{+} \times \mathbb{R}_{+} \rightarrow \mathbb{R}_{+}$:

$$
Y_{t}=z_{t} F\left(K_{t}, N_{t}\right)+(1-\delta) K_{t}
$$

where aggregate capital $K_{t}$ is $\mathcal{F}_{t-1}$-measurable, aggregate labor $N_{t}$ is $\mathcal{F}_{t}$-measurable, and $\delta \in$ $(0,1)$ is the depreciation rate. Capital is transformed from consumers' accumulated assets and aggregate labor supply $N_{t}$ is given exogenously.

Assumption 5 (a) $N_{t}$ is uniformly bounded, $0 \leq N_{t} \leq \widehat{N}$. (b) $F$ is homogeneous of degree one, strictly increasing, strictly concave, continuously differentiable, and satisfies: $F(0, \cdot)=F(\cdot, 0)=$ $0, \lim _{K \rightarrow 0} F_{1}(K, \widehat{N})=\infty$, and $\lim _{K \rightarrow \infty} F_{1}(K, \widehat{N})=0$.

Remark 2.2 This assumption implies that there is a maximal sustainable capital stock $\widehat{K}$ which is given by the unique solution to the equation $\bar{z} F(K, \widehat{N})=\delta K$.

By Assumption 5, competitive profit maximization implies that for all $t \geq 0$,

$$
\begin{aligned}
r_{t} & =z_{t} F_{1}\left(K_{t}, N_{t}\right)-\delta, \\
w_{t} & =z_{t} F_{2}\left(K_{t}, N_{t}\right) .
\end{aligned}
$$

\footnotetext{
${ }^{8}$ The proof of existence of admissible allocations follows from a similar argument in [21]. So I omit it.
} 
Note that prices $r_{t}$ and $w_{t}$ are $\mathcal{F}_{t}$-measurable.

\subsection{Competitive Equilibrium}

I first define sequential competitive equilibrium in the standard way.

Definition 1. A sequential competitive equilibrium $\left(\left(\left(a_{t+1}^{i}, c_{t}^{i}\right)_{t \geq 0}\right)_{i \in I},\left(r_{t}, w_{t}\right)_{t \geq 0}\right)$ consists of an admissible allocation $\left(\left(a_{t+1}^{i}, c_{t}^{i}\right)_{t \geq 0}\right)_{i \in I}$ and price processes $\left(r_{t}, w_{t}\right)_{t \geq 0}$ such that: (i) Given prices $\left(w_{t}, r_{t}\right)_{t \geq 0},\left(a_{t+1}^{i}, c_{t}^{i}\right)_{t \geq 0}$ solves problem (3) for $\phi$-a.e. $i$. (ii) Given prices $\left(w_{t}, r_{t}\right)_{t \geq 0}$, the firm maximizes profits so that (4) and (5) are satisfied for all $t \geq 0$. (iii) Markets clear, i.e., for all $t \geq 0$,

$$
\begin{gathered}
\int_{I} s_{t}^{i} \phi(d i)=N_{t} \\
C_{t}+K_{t+1}=z_{t} F\left(K_{t}, N_{t}\right)+(1-\delta) K_{t},
\end{gathered}
$$

where $C_{t}=\int_{I} c_{t}^{i} \phi(d i)$ and $K_{t}=\int_{I} a_{t}^{i} \phi(d i)$.

To analyze the existence and properties of sequential competitive equilibria, it is important to introduce the notion of aggregate distribution. Such a distribution is defined over the individual states across the population. An individual state is a pair of individual asset holdings and the history of individual shocks. More formally, if individual asset holdings and the shock history at date $t \geq 0$ are $a_{t}^{i}$ and $s^{t i}$, respectively, $i \in I$, then the aggregate distribution, $\lambda_{t} \in \mathcal{P}\left(\mathbb{A} \times \mathbb{S}^{t}\right)$, is defined by:

$$
\lambda_{t}(A \times B)=\phi\left(i \in I:\left(a_{t}(i), s^{t}(i)\right) \in A \times B\right), A \times B \in \mathcal{B}(\mathbb{A}) \times \mathcal{B}\left(\mathbb{S}^{t}\right) .
$$

Thus, $\lambda_{t}(A \times B)$ is the measure of consumers whose asset holdings and shock histories at date $t$ lie in the set $A \times B$. Note that $\lambda_{t}$ is a random measure since $a_{t}^{i}=a_{t}^{i}\left(\omega, z^{t-1}\right)$ and $s_{t}^{i}=s_{t}^{i}\left(\omega, z^{t}\right)$ are random variables.

Any aggregate variable can be written as an expectation with respect to the so defined aggregate distribution; for example,

$$
\begin{aligned}
K_{t} & =\int_{I} a_{t}^{i} \phi(d i)=\int_{\mathbb{A} \times \mathbb{S}^{t}} a \lambda_{t}\left(d a, d s^{t}\right), \\
N_{t} & =\int_{I} s_{t}^{i} \phi(d i)=\int_{\mathbb{A} \times \mathbb{S}^{t}} s \lambda_{t}\left(d a, d s^{t}\right), \\
C_{t} & =\int_{I} c_{t}^{i} \phi(d i)=\left(1+r_{t}\right) K_{t}+w_{t} N_{t}-K_{t+1} .
\end{aligned}
$$


The last equation follows from integration of Eq. (1). It implies the resource constraint (7) by the homogeneity of $F$ and (4)-(5). Finally, Eq. (4)-(5) induce pricing functions $r_{t}$ : $\mathcal{P}\left(\mathbb{A} \times \mathbb{S}^{t}\right) \times \mathbb{Z} \rightarrow \mathbb{R}$ and $w_{t}: \mathcal{P}\left(\mathbb{A} \times \mathbb{S}^{t}\right) \times \mathbb{Z} \rightarrow \mathbb{R}_{+}$as follows:

$$
\begin{aligned}
r_{t}\left(\lambda_{t}, z_{t}\right) & =z_{t} F_{1}\left(\int_{\mathbb{A} \times \mathbb{S}^{t}} a \lambda_{t}\left(d a, d s^{t}\right), \int_{\mathbb{A} \times \mathbb{S}^{t}} s \lambda_{t}\left(d a, d s^{t}\right)\right)-\delta, \\
w_{t}\left(\lambda_{t}, z_{t}\right) & =z_{t} F_{2}\left(\int_{\mathbb{A} \times \mathbb{S}^{t}} a \lambda_{t}\left(d a, d s^{t}\right), \int_{\mathbb{A} \times \mathbb{S}^{t}} s \lambda_{t}\left(d a, d s^{t}\right)\right) .
\end{aligned}
$$

From the above discussion, conclude that aggregate distributions contain all the relevant information for equilibrium analysis. Henceforth, they will be the focus of study.

\section{EXISTENCE OF COMPETITIVE EQUILIBRIUM}

I begin by analyzing a single consumer's decision problem. I then discuss aggregation. I finally present the existence result. Notice that the model reduces to the case without aggregate shocks when $\mathbb{Z}$ contains only one element. Thus, all results to follow are valid for this case. ${ }^{9}$

\subsection{The One-Person Decision Problem}

Consider a single consumer's decision problem, given a sequence of aggregate distributions $\mu=$ $\left\{\lambda_{t}\right\}_{t \geq 0}$. So the consumer index is suppressed.

In general, the aggregate distribution at date $t$ is a measurable function of the individualrelevant state $\omega$ and the history of aggregate shocks $z^{t}$ (see (8)). However, Section 3.2 will show that under some conditions, equilibrium aggregate distributions do not depend on the individual-relevant state $\omega$. Therefore, this subsection assumes that the aggregate distribution $\lambda_{t}$ is a function from the set of histories of aggregate shocks $\mathbb{Z}^{t}$ to $\mathcal{P}\left(\mathbb{A} \times \mathbb{S}^{t}\right)$. Let $\mathcal{P}\left(\mathbb{A} \times \mathbb{S}^{t}\right)^{\mathbb{Z}^{t}}$ denote the set of such functions endowed with the product (or pointwise convergence) topology. Let $\mathcal{P}_{\infty}(\mathbb{A} \times \mathbb{S}) \equiv \times_{t=0}^{\infty} \mathcal{P}\left(\mathbb{A} \times \mathbb{S}^{t}\right)^{\mathbb{Z}^{t}}$. Then $\mu$ is an element in $\mathcal{P}_{\infty}(\mathbb{A} \times \mathbb{S})$.

It is convenient to analyze an individual's consumption and savings decisions by dynamic programming. Let $V_{t}\left(a_{t}, s^{t}, z^{t}, \mu\right)$ denote the maximized expected discounted utility of the consumer at date $t$, when his asset holdings are $a_{t}$ and the sequence of aggregate distributions is $\mu$, given the individual shock history $s^{t}$ and the aggregate shock history $z^{t}$. Then, at date $t \geq 0$,

\footnotetext{
${ }^{9}$ Aiyagari [2] and Miao [31] study stationary equilibria for economies without aggregate shocks.
} 
the consumer solves the following dynamic programming problem:

$$
\begin{aligned}
V_{t}\left(a_{t}, s^{t}, z^{t}, \mu\right)= & \sup _{a_{t+1} \in \Gamma\left(a_{t}, s_{t}, z_{t}, \lambda_{t}\left(z^{t}\right)\right)} u\left(\left(1+r_{t}\left(\lambda_{t}\left(z^{t}\right), z_{t}\right)\right) a_{t}+w_{t}\left(\lambda_{t}\left(z^{t}\right), z_{t}\right) s_{t}-a_{t+1}\right) \\
& +\beta \int_{\mathbb{S} \times \mathbb{Z}} V_{t+1}\left(a_{t+1}, s^{t+1}, z^{t+1}, \mu\right) Q_{t+1}\left(d s_{t+1}, d z_{t+1}, s^{t}, z^{t}\right),
\end{aligned}
$$

where

$$
\Gamma\left(a_{t}, s_{t}, z_{t}, \lambda_{t}\left(z^{t}\right)\right)=\left[0,\left(1+r_{t}\left(\lambda_{t}\left(z^{t}\right), z_{t}\right)\right) a_{t}+w_{t}\left(\lambda_{t}\left(z^{t}\right), z_{t}\right) s_{t}\right] .
$$

The associated policy correspondence is defined by $g_{t+1}: \mathbb{A} \times \mathbb{S}^{t} \times \mathbb{Z}^{t} \times \mathcal{P}_{\infty}(\mathbb{A} \times \mathbb{S}) \rightarrow \mathbb{A}$, with $g_{t+1}\left(a_{t}, s^{t}, z^{t}, \mu\right) \subset \Gamma\left(a_{t}, s_{t}, z_{t}, \lambda_{t}\left(z^{t}\right)\right)$. If $g_{t+1}$ is single-valued, it is called a policy function. If $g_{t+1}\left(a_{t}, s^{t}, z^{t}, \mu\right)$ is the set of maximizers of problem (11), it is called an optimal policy correspondence.

To understand problem (11), consider an $n$-period truncation. At date $n$, the consumer solves the following problem:

$$
V_{n}^{n}\left(a_{n}, s_{n}, z^{n}, \lambda_{n}\left(z^{n}\right)\right)=\max _{a^{\prime} \in \Gamma\left(a_{n}, s_{n}, z_{n}, \lambda_{n}\left(z^{n}\right)\right)} u\left(\left(1+r_{n}\left(\lambda_{n}\left(z^{n}\right), z_{n}\right) a_{n}+w_{n}\left(\lambda_{n}\left(z^{n}\right), z_{n}\right) s_{n}-a^{\prime}\right) .\right.
$$

At date $n-1$, by the principle of optimally, the consumer solves the following problem:

$$
\begin{aligned}
& V_{n-1}^{n}\left(a_{n-1}, s^{n-1}, z^{n-1}, \lambda_{n-1}\left(z^{n-1}\right), \lambda_{n}\right)=\underset{a^{\prime} \in \Gamma\left(a_{n-1}, s_{n-1}, z_{n-1}, \lambda_{n-1}\left(z^{n-1}\right)\right)}{\max } \\
& u\left(\left(1+r_{n-1}\left(\lambda_{n-1}\left(z^{n-1}\right), z_{n-1}\right) a_{n-1}+w_{n-1}\left(\lambda_{n-1}\left(z^{n-1}\right), z_{n-1}\right) s_{n-1}-a^{\prime}\right)\right. \\
& +\beta \int_{\mathbb{S} \times \mathbb{Z}} V_{n}^{n}\left(a^{\prime}, s_{n}, z^{n}, \lambda_{n}\left(z^{n}\right)\right) Q_{n}\left(d z_{n}, d s_{n}, s^{n-1}, z^{n-1}\right) .
\end{aligned}
$$

In general, at any date $0 \leq t \leq n$, the consumer solves the problem:

$$
\begin{aligned}
& V_{t}^{n}\left(a_{t}, s^{t}, z^{t}, \lambda_{t}\left(z^{t}\right), \lambda_{t+1}, \ldots, \lambda_{n}\right) \\
= & \max _{a^{\prime} \in \Gamma\left(a_{t}, s_{t}, z_{t}, \lambda_{t}\left(z^{t}\right)\right)} u\left(\left(1+r_{t}\left(\lambda_{t}\left(z^{t}\right), z_{t}\right) a_{t}+w_{t}\left(\lambda_{t}\left(z^{t}\right), z_{t}\right) s_{t}-a^{\prime}\right)\right. \\
& +\beta \int_{\mathbb{S} \times \mathbb{Z}} V_{t+1}^{n}\left(a^{\prime}, s^{t+1}, z^{t+1}, \lambda_{t+1}\left(z^{t+1}\right), \lambda_{t+2}, \ldots, \lambda_{n}\right) Q_{t+1}\left(d z_{t+1}, d s_{t+1}, s^{t}, z^{t}\right) .
\end{aligned}
$$

Problem (11) corresponds to the limiting case as $n \rightarrow \infty$.

More formally, let $\mathbb{V}$ denote the set of uniformly bounded and continuous real-valued functions on $\mathbb{A} \times \mathbb{S}^{t} \times \mathbb{Z}^{t} \times \mathcal{P}_{\infty}(\mathbb{A} \times \mathbb{S})$. Let $\mathbb{V}^{\infty}$ denote the set of sequences $v=\left(v_{0}, v_{1}, v_{2}, \ldots\right)$ of such functions. Note that $\mathbb{V}^{\infty}$ is a complete metric space if endowed with the norm

$$
\|v\|=\sup _{\left(t, a_{t}, s^{t}, z^{t}, \mu\right)}\left|v_{t}\left(a_{t}, s^{t}, z^{t}, \mu\right)\right| .
$$


Then an application of the Contraction Mapping Theorem yields:

Lemma 1. Given Assumptions 1-5, then there is a unique sequence of functions $\left\{V_{t}\right\}_{t \geq 0} \in \mathbb{V}^{\infty}$ and a unique sequence of continuous policy functions $\left\{g_{t+1}\right\}_{t \geq 0}$ solving (11).

\subsection{Aggregation and the Law of Motion for Aggregate Distributions}

This subsection studies the question of aggregation of individual behavior to form aggregate behavior and derives the law of motion for the aggregate distributions induced by the sequences of individual optimal policy functions $\left\{g_{t+1}\right\}_{t \geq 0}$ and individual shocks $\left(s_{t}^{i}\right)_{t \geq 0}$.

In perfectly competitive markets, each consumer has no influence over prices, and all consumers together determine prices. The continuum formulation and a suitable law of large numbers make this possible. To see this, recall that the aggregate distribution at date $t, \lambda_{t}\left(\omega, z^{t}\right)$, is defined in (8). It is a random measure that depends on the state $\left(\omega, z^{t}\right)$. In models without aggregate shocks (e.g., [2] and [31]), perfect competition implies that equilibrium aggregate distributions must be deterministic. The latter can be achieved by assuming a no aggregate uncertainty condition on the shock processes and the underlying probability spaces, introduced in [6, Definition 1] for models of anonymous sequential games. Feldman and Gilles' construction [13, Proposition 2 ] shows that this condition is not vacuous and their construction is applied directly by Miao [31] to a Bewley-style model without aggregate shocks.

Say that a process $X=\left(X_{t}\right)_{t \geq 0}, X_{t}: I \times \Omega \rightarrow \mathbb{D}$, where $\mathbb{D}$ is a Euclidean space and $X_{t}$ is jointly measurable, satisfies no aggregate uncertainty if there exists a nonrandom measure $\nu$ such that $\phi(i \in I: X(i, \omega) \in D)=\nu(D), D \in \mathcal{B}(\mathbb{D})$, for $P$-a.e. $\omega{ }^{10}$ Note that whether or not a process $X$ has the no aggregate uncertainty property depends on the underlying probability space. The implication of the no aggregate uncertainty condition is that $\phi(i \in I: X(i, \omega) \in$ $D)=P(\omega \in \Omega: X(i, \omega) \in D)$ if each $X^{i}$ is drawn from the same distribution. In this case, the measure $\nu$ is in fact this common distribution. Thus, the empirical distribution of a sample of random variables $\left(X_{t}^{i}\right)_{i \in I}$ is the same as the theoretical distribution from which all these random variables are drawn.

To accommodate the case where aggregate shocks are present, I follow [6] and introduce a notion of conditional no aggregate uncertainty. A process $X=\left(X_{t}\right)_{t \geq 0}, X_{t}: I \times \Omega \times \mathbb{Z}^{\infty} \rightarrow \mathbb{D}$,

\footnotetext{
${ }^{10}$ Note that this definition is slightly different from [6, Definition 1].
} 
satisfies the conditional no aggregate uncertainty condition if given the history of aggregate shocks $z^{\infty} \in \mathbb{Z}^{\infty}, X$ satisfies the no aggregate uncertainty condition. I now assume:

Assumption 6 The individual shock process $s^{i}=\left(s_{t}^{i}\right)_{t \geq 0}, s_{t}: I \times \Omega \times \mathbb{Z}^{\infty} \rightarrow \mathbb{S}$, satisfies the conditional no aggregate uncertainty condition relative to the probability space $\left(\Omega \times \mathbb{Z}^{\infty}, \mathcal{F}, P\right)$.

This assumption implies that given the history $z^{\infty}$,

$$
\phi\left(i \in I: s\left(i, \omega, z^{\infty}\right) \in B\right)=P_{z}\left(\omega \in \Omega: s^{i}\left(\omega, z^{\infty}\right) \in B\right), B \in \mathcal{B}\left(\mathbb{S}^{\infty}\right)
$$

where $P_{z}$ is the conditional measure on $\Omega$ given $z^{\infty}$. Thus, conditional on the history of aggregate shocks $z^{t}$, aggregate labor endowments satisfy

$$
\int_{I} s_{t}^{i} \phi(d i)=\int_{\mathbb{A} \times \mathbb{S}^{t}} s \lambda_{t}\left(d a, d s^{t}\right)=\int_{\Omega} s_{t}^{i}\left(\omega, z^{t}\right) P_{z}(d \omega), \forall t \geq 0, \forall i \in I
$$

which is deterministic. This property, along with the labor market clearing condition (6), puts a restriction on aggregate labor supply $N_{t}$; namely, $N_{t}$ must depend on $z^{t}$ only.

Assumption 6 permits derivation of the law of motion for aggregate distributions, as I now show. Because consumers are ex ante identical, they will choose the same optimal asset accumulation policy. Thus, given the individual state $\left(a_{t}^{i}, s^{t i}\right)$, the history of aggregate shocks $z^{t}$, and the sequence of aggregate distributions $\mu$, let the asset holdings next period be $a_{t+1}^{i}=g_{t+1}\left(a_{t}^{i}, s^{t i}, z^{t}, \mu\right)$ for $\phi$-a.e. $i$.

Fixing a history of shocks $z^{t+1}$ and using (8) and Bayes' Rule, one can derive that for any Borel sets $A \in \mathcal{B}(\mathbb{A}), B=B_{1} \times B_{2} \in \mathcal{B}\left(\mathbb{S}^{t}\right) \times \mathcal{B}(\mathbb{S})$,

$$
\begin{gathered}
\lambda_{t+1}\left(\omega, z^{t+1}\right)(A \times B)=\phi\left(i \in I:\left(a_{t+1}(i), s^{t+1}(i)\right) \in A \times B\right) \\
=\int_{\mathbb{A} \times B_{1}} \phi\left(i \in I:\left(g_{t+1}\left(a_{t}^{i}, s^{t i}, z^{t}, \mu\right), s^{t+1, i}\right) \in A \times B \mid\left(a_{t}^{i}, s^{t i}\right)=\left(a_{t}, s^{t}\right)\right) \\
\cdot \phi\left(i \in I:\left(a_{t}^{i}, s^{t i}\right) \in d a_{t} \times d s^{t}\right) . \\
=\int_{\mathbb{A} \times B_{1}} \phi\left(i \in I:\left(g_{t+1}\left(a_{t}, s^{t}, z^{t}, \mu\right), s_{t+1}^{i}\left(z^{t+1}\right)\right) \in A \times B_{2} \mid s^{t i}=s^{t}\right) \lambda_{t}\left(d a_{t}, d s^{t}\right) \\
=\int_{\mathbb{A} \times B_{1}} \mathbf{1}_{A}\left(g\left(a, s^{t}, z^{t}, \mu\right)\right) \phi\left(i \in I: s_{t+1}\left(i, \omega, z^{t+1}\right) \in B_{2} \mid s^{t i}=s^{t}\right) \lambda_{t}\left(d a_{t}, d s^{t}\right)
\end{gathered}
$$

Applying the conditional no aggregate uncertainty condition, one obtains:

$$
\phi\left(i \in I: s_{t+1}\left(i, \omega, z^{t+1}\right) \in B_{2} \mid s^{t i}=s^{t}\right)=Q_{t+1}\left(B_{2}, z_{t+1}, s^{t}, z^{t}\right) .
$$


This implies that, conditional on the history of aggregate shocks $z^{t+1}$ and the history of individual shocks $s^{t}, \phi\left(i \in I: s_{t+1}\left(i, \omega, z^{t+1}\right) \in B_{2} \mid s^{t i}=s^{t}\right)$ does not depend on individual uncertainty. Therefore, if $\lambda_{0}$ is a nonrandom measure, then the conditional no aggregate uncertainty condition implies that, conditional on any history of aggregate shocks, the aggregate distribution at each date does not depend on individual uncertainty. Thus, the date $t$ aggregate distribution $\lambda_{t}$ can be identified as a map from $\mathbb{Z}^{t}$ to $\mathcal{P}\left(\mathbb{A} \times \mathbb{S}^{t}\right)$, as assumed in Section 3.1.

The above discussion is summarized in the following Lemma:

Lemma 2. Under the conditional no aggregate uncertainty condition Assumption 6, along a history of aggregate shocks $z^{\infty}=\left(z_{0}, z_{1}, \ldots\right)$, the sequence of equilibrium aggregate distributions $\mu=\left(\lambda_{t}\right)_{t \geq 0}$ evolves according to

$$
\lambda_{t+1}\left(z^{t+1}\right)(A \times B)=\int_{\mathbb{A} \times B_{1}} \mathbf{1}_{A}\left(g_{t+1}\left(a_{t}, s^{t}, z^{t}, \mu\right)\right) Q_{t+1}\left(B_{2}, z_{t+1}, s^{t}, z^{t}\right) \lambda_{t}\left(d a_{t}, d s^{t}\right)\left(z^{t}\right),
$$

for any Borel sets $A \in \mathcal{B}(\mathbb{A})$ and $B=B_{1} \times B_{2} \in \mathcal{B}\left(\mathbb{S}^{t}\right) \times \mathcal{B}(\mathbb{S})$.

\subsection{The Existence Theorem}

I now state one main result of the paper.

Theorem 1. Given Assumptions 1-6, there exists a sequential competitive equilibrium. Moreover, the set of equilibrium sequences of aggregate distributions are compact.

The idea of the proof can be described as follows. Consider a sequence of aggregate distributions $\mu=\left(\lambda_{t}\left(z^{t}\right)\right)_{t \geq 0} \in \mathcal{P}_{\infty}(\mathbb{A} \times \mathbb{S})$ along a history of aggregate shocks $z^{\infty}$. Denote by $\mathcal{P}_{\infty}^{0}(\mathbb{A} \times \mathbb{S})$ the set of all such sequences satisfying the labor market clearing condition

$$
\int_{\mathbb{A} \times \mathbb{S}^{t}} s \lambda_{t}\left(d a, d s^{t}\right)=N_{t}, t \geq 0
$$

A sequence of optimal asset accumulation policies $\left\{g_{t+1}\right\}_{t \geq 0}$ can be derived from Lemma 1. Define a new sequence of aggregate distributions $\widetilde{\mu}=\left(\widetilde{\lambda}_{t}\left(z^{t}\right)\right)_{t \geq 0}$ by: $\widetilde{\lambda}_{0}\left(z^{0}\right)=\lambda_{0}\left(z_{0}\right)$,

$$
\widetilde{\lambda}_{t+1}\left(z^{t+1}\right)(A \times B)=\int_{\mathbb{A} \times B_{1}} \mathbf{1}_{A}\left(g_{t+1}\left(a_{t}, s^{t}, z^{t}, \mu\right)\right) Q_{t+1}\left(B_{2}, z_{t+1}, s^{t}, z^{t}\right) \lambda_{t}\left(d a_{t}, d s^{t}\right),
$$

for all Borel sets $A \in \mathcal{B}(\mathbb{A})$ and $B=B_{1} \times B_{2} \in \mathcal{B}\left(\mathbb{S}^{t}\right) \times \mathcal{B}(\mathbb{S}), t \geq 0$. Furthermore, define a $\operatorname{map} \psi: \mathcal{P}_{\infty}^{0}(\mathbb{A} \times \mathbb{S}) \rightarrow \mathcal{P}_{\infty}^{0}(\mathbb{A} \times \mathbb{S})$ by $\psi(\mu)=\widetilde{\mu}$. Then the fixed point of $\psi, \mu^{*}=\left(\lambda_{0}^{*}, \lambda_{1}^{*}, \lambda_{2}^{*}, \ldots\right)$, 
induces a sequential competitive equilibrium $\left(\left(\left(a_{t+1}^{i}, c_{t}^{i}\right)_{t \geq 0}\right)_{i \in I},\left(r_{t}, w_{t}\right)_{t \geq 0}\right)$. Specifically, for any histories of shocks $\left(s^{t i}, z^{t}\right)$, let

$$
\begin{aligned}
a_{t+1}^{i} & =g_{t+1}\left(a_{t}^{i}, s_{t}^{i}, z_{t},\left(\lambda_{\tau}^{*}\right)_{\tau \geq t}\right), c_{t}^{i}=\left(1+r_{t}\right) a_{t}^{i}+w_{t} s_{t}^{i}-a_{t+1}^{i}, \\
r_{t} & =z_{t} F_{1}\left(K_{t}, N_{t}\right)-\delta, w_{t}=z_{t} F_{2}\left(K_{t}, N_{t}\right), \\
K_{t} & =\int_{\mathbb{A} \times \mathbb{S}^{t}} a \lambda_{t}^{*}\left(d a, d s^{t}\right), \int_{\mathbb{A} \times \mathbb{S}^{t}} s \lambda_{t}^{*}\left(d a, d s^{t}\right)=N_{t},
\end{aligned}
$$

where $a_{0}^{i}, s_{0}^{i}, z_{0}$, and $\lambda_{0}^{*}=\lambda_{0}$ are given.

However, $\mathcal{P}_{\infty}^{0}(\mathbb{A} \times \mathbb{S})$ is not a compact set since $\mathbb{A}$ is not compact. To apply the BrouwerSchauder-Tychonoff Fixed-Point Theorem [3, Corollary 16.52], one needs the domain of $\psi$ to be compact. Thus, I construct another compact set so that $\psi$ is a self-map in this domain.

The set is constructed as follows. Because of Assumption 5 and the resource constraint, one can restrict attention to the set of sequences of aggregate distributions $\left(\lambda_{t}\right)_{t \geq 0}$ such that $K_{t}=\int_{\mathbb{A} \times \mathbb{S}^{t}} a \lambda_{t}\left(d a, d s^{t}\right) \leq \widehat{K}$. Then let

$$
\begin{gathered}
\widehat{\mathcal{P}}\left(\mathbb{A} \times \mathbb{S}^{t}\right)\left(z^{t}\right)=\left\{\lambda\left(z^{t}\right) \in \mathcal{P}\left(\mathbb{A} \times \mathbb{S}^{t}\right): \int_{\mathbb{A} \times \mathbb{S}^{t}} a \lambda\left(z^{t}\right)\left(d a, d s^{t}\right) \leq \widehat{K}, \int_{\mathbb{A} \times \mathbb{S}^{t}} s \lambda\left(z^{t}\right)\left(d a, d s^{t}\right)=N_{t}\left(z^{t}\right)\right\}, \\
\widehat{\mathcal{P}}_{\infty}(\mathbb{A} \times \mathbb{S})=\times_{t=0}^{\infty} \times_{z^{t} \in \mathbb{Z}^{t}} \widehat{\mathcal{P}}\left(\mathbb{A} \times \mathbb{S}^{t}\right)\left(z^{t}\right) .
\end{gathered}
$$

Lemma 3. $\widehat{\mathcal{P}}_{\infty}(A \times S)$ is a compact and convex subset of a locally convex Hausdorff space.

One can now apply the Brouwer-Schauder-Tychonoff Fixed-Point Theorem to the map $\psi$ : $\widehat{\mathcal{P}}_{\infty}(\mathbb{A} \times \mathbb{S}) \rightarrow \widehat{\mathcal{P}}_{\infty}(\mathbb{A} \times \mathbb{S})$. Any fixed point induces a competitive equilibrium.

\section{RECURSIVE CHARACTERIZATION}

To permit a recursive characterization of sequential competitive equilibria, I make two stationarity assumptions:

Assumption $7 Q_{t+1}\left(S \times Z, s^{t}, z^{t}\right)=Q\left(S \times Z, s_{t}, z_{t}\right)$ for all $t \geq 0$ and $S \times Z \in \mathcal{B}(\mathbb{S}) \times \mathcal{B}(\mathbb{Z})$.

Assumption 8 Aggregate labor endowments at any date $t \geq 0$ is given by a measurable function $N: \mathbb{Z}^{t} \rightarrow(0, \widehat{N}]$. 
Given these assumptions, the economy is the same as that studied by Krusell and Smith [24]. These two assumptions also imply that past histories of individual shocks do not affect current decisions. Thus, the aggregate distribution of asset holdings and individual shocks at date $t, \lambda_{t}$, can be defined as

$$
\lambda_{t}(A \times B)=\phi\left(i \in I:\left(a_{t}^{i}, s_{t}^{i}\right) \in A \times B\right), A \times B \in \mathcal{B}(\mathbb{A}) \times \mathcal{B}(\mathbb{S}) .
$$

The set of all aggregate distributions is denoted by $\mathcal{P}_{\infty}(\mathbb{A} \times \mathbb{S})=\times_{t=0}^{\infty} \mathcal{P}(\mathbb{A} \times \mathbb{S})^{\mathbb{Z}^{t}}$.

Under Assumptions 1-8, the pricing functions (4)-(5) become $r: \mathcal{P}(\mathbb{A} \times \mathbb{S}) \times \mathbb{Z} \rightarrow \mathbb{R}, w$ : $\mathcal{P}(\mathbb{A} \times \mathbb{S}) \times \mathbb{Z} \rightarrow \mathbb{R}_{+}$,

$$
\begin{aligned}
& r(\lambda, z)=z F_{1}\left(\int_{\mathbb{A} \times \mathbb{S}} a \lambda(d a, d s), \int_{\mathbb{A} \times \mathbb{S}} s \lambda(d a, d s)\right)-\delta, \\
& w(\lambda, z)=z F_{2}\left(\int_{\mathbb{A} \times \mathbb{S}} a \lambda(d a, d s), \int_{\mathbb{A} \times \mathbb{S}} s \lambda(d a, d s)\right) .
\end{aligned}
$$

Moreover, a typical consumer's decision problem at date $t(11)$ can be rewritten as the following dynamic programming equation:

$$
\begin{aligned}
V\left(a_{t}, s_{t}, z_{t},\left(\lambda_{\tau}\right)_{\tau \geq t}\right)= & \sup _{a^{\prime} \in \Gamma\left(a_{t}, s_{t}, z_{t}, \lambda_{t}\right)} u\left(\left(1+r\left(\lambda_{t}, z_{t}\right)\right) a_{t}+w\left(\lambda_{t}, z_{t}\right) s_{t}-a^{\prime}\right) \\
& +\beta \int_{\mathbb{S} \times \mathbb{Z}} V\left(a^{\prime}, s^{\prime}, z^{\prime},\left(\lambda_{\tau}\right)_{\tau \geq t+1}\right) Q\left(d s^{\prime}, d z^{\prime}, s_{t}, z_{t}\right) .
\end{aligned}
$$

This problem is studied in Lemma 4 below.

To derive a recursive characterization, it is important to select state variables. A current state must be a sufficient statistic for the future evolution of the system. With incomplete markets and heterogeneous consumers, equilibrium prices generally depend on the distribution of assets across the consumers. Thus, it is natural to include the aggregate distribution as a state variable. The question is whether it constitutes a sufficient endogenous aggregate state. To answer this question, I define a notion of equilibrium correspondence in the next subsection.

\subsection{Equilibrium Correspondence}

I first provide a lemma characterizing an equilibrium sequence of aggregate distributions.

Lemma 4. Let Assumptions 1-8 hold. Then:

(i) There is a unique continuous and bounded function $V: \mathbb{A} \times \mathbb{S} \times \mathbb{Z} \times \mathcal{P}_{\infty}(\mathbb{A} \times \mathbb{S}) \rightarrow \mathbb{R}$ and a unique continuous policy function $g: \mathbb{A} \times \mathbb{S} \times \mathbb{Z} \times \mathcal{P}_{\infty}(\mathbb{A} \times \mathbb{S}) \rightarrow \mathbb{A}$ solving problem (16). 
(ii) Any equilibrium sequence of aggregate distributions $\left(\lambda_{t}\right)_{t \geq 0}$ is characterized by the following equations: for $t \geq 0, A \times B \in \mathcal{B}(\mathbb{A}) \times \mathcal{B}(\mathbb{S})$,

$$
\begin{gathered}
\int_{\mathbb{A} \times \mathbb{S}} s \lambda_{t}\left(z^{t}\right)(d a, d s)=N\left(z^{t}\right), \\
\lambda_{t+1}\left(z^{t+1}\right)(A \times B)=\int_{\mathbb{A} \times \mathbb{S}} \mathbf{1}_{A}\left(g\left(a_{t}, s_{t}, z_{t},\left(\lambda_{\tau}\right)_{\tau \geq t}\right)\right) Q\left(B, z_{t+1}, s_{t}, z_{t}\right) \lambda_{t}\left(z^{t}\right)\left(d a_{t}, d s_{t}\right),
\end{gathered}
$$

where $\lambda_{0}$ is given.

Eq. (17) is the labor market clearing condition. Eq. (18) says that the evolution of $\left(\lambda_{t}\right)_{t \geq 0}$ must be consistent with consumers' optimal behavior. It embodies rational expectations.

I now define an equilibrium correspondence $\mathcal{E}: \mathbb{Z} \times \mathcal{P}(\mathbb{A} \times \mathbb{S}) \rightarrow \mathcal{P}_{\infty}(\mathbb{A} \times \mathbb{S})$, where $\mathcal{E}(z, \lambda)$ is the set of equilibrium sequences of aggregate distributions associated with an initial aggregate state $(z, \lambda)$. Theorem 1 shows that $\mathcal{E}(z, \lambda)$ is nonempty and compact so that the correspondence $\mathcal{E}$ is well defined. An important property of the equilibrium correspondence is described in the following lemma.

Lemma 5. Under Assumptions 1-8, the equilibrium correspondence $\mathcal{E}$ is upper hemicontinuous.

Because the equilibrium correspondence is generally not single-valued, there may be multiple equilibrium trajectories that are consistent with a given initial aggregate distribution and a given initial value of aggregate shock. This implies that the current aggregate distribution is typically not a sufficient (endogenous) statistic for the future evolution of the aggregate distributions (or prices). This motivates the need for additional state variables.

Before I turn to recursive characterizations in the next subsection, I define another correspondence. Let

$$
\begin{aligned}
\mathbb{X} & =\{(z, \lambda, v) \in \mathbb{Z} \times \mathcal{P}(\mathbb{A} \times \mathbb{S}) \times \mathbb{C}(\mathbb{A} \times \mathbb{S} \times \mathbb{Z} \times \mathcal{P}(\mathbb{A} \times \mathbb{S})): \\
\exists \mu & \in \mathcal{E}(z, \lambda), v(\cdot, z, \lambda)=V(\cdot, z, \mu))\}
\end{aligned}
$$

Define a correspondence $\varphi: \mathbb{X} \rightarrow \mathcal{P}_{\infty}(\mathbb{A} \times \mathbb{S})$ as

$$
\varphi(z, \lambda, v)=\{\mu \in \mathcal{E}(z, \lambda): v(\cdot, z, \lambda)=V(\cdot, z, \mu)\}
$$

Thus, the correspondence $\varphi$ assigns to any point $(z, \lambda, v) \in \mathbb{X}$ the set of equilibrium sequences of aggregate distributions $\mu$ with the property that the expected payoff to consumer $i$ is $v(a, s, z, \lambda)$ 
when the initial state is $\left(a_{0}^{i}, s_{0}^{i}, z_{0}, \lambda_{0}\right)=(a, s, z, \lambda)$. Since $\mathcal{E}(z, \lambda)$ is nonempty by Theorem 1 , the correspondence $\varphi$ is well defined. Using Lemma 5, one can establish the following lemma, which is important for the recursive characterization studied in the next subsection.

Lemma 6. Under Assumptions 1-8, the correspondence $\varphi$ is upper hemicontinuous.

\subsection{Recursive Equilibria}

I now turn to recursive characterization of sequential competitive equilibria. The key to recursive characterization is the selection of state variables. Inspired by the literature on sequential games $[11,5,7]$ and on dynamic contracts $[32,36,1]$, I include the expected payoffs (expected discounted utilities) as an additional endogenous state variable and define a recursive equilibrium as follows.

Definition 2. A recursive (competitive) equilibrium $\left(\left(f, T^{v}, G\right),(r, w)\right)$ consists of a measurable policy function $f: \mathbb{A} \times \mathbb{S} \times \mathbb{Z} \times \mathcal{P}(\mathbb{A} \times \mathbb{S}) \times \mathbb{C}(\mathbb{A} \times \mathbb{S} \times \mathbb{Z} \times \mathcal{P}(\mathbb{A} \times \mathbb{S})) \rightarrow \mathbb{A}$, a measurable map $T^{v}: \mathbb{Z} \times \mathcal{P}(\mathbb{A} \times \mathbb{S}) \times \mathbb{C}(\mathbb{A} \times \mathbb{S} \times \mathbb{Z} \times \mathcal{P}(\mathbb{A} \times \mathbb{S})) \rightarrow \mathbb{C}(\mathbb{A} \times \mathbb{S} \times \mathbb{Z} \times \mathcal{P}(\mathbb{A} \times \mathbb{S}))$, a measurable map $G: \mathbb{Z} \times \mathcal{P}(\mathbb{A} \times \mathbb{S}) \times \mathbb{C}(\mathbb{A} \times \mathbb{S} \times \mathbb{Z} \times \mathcal{P}(\mathbb{A} \times \mathbb{S})) \times \mathbb{Z} \rightarrow \mathcal{P}(\mathbb{A} \times \mathbb{S})$, and measurable pricing functions $r: \mathcal{P}(\mathbb{A} \times \mathbb{S}) \times \mathbb{Z} \rightarrow \mathbb{R}$ and $w: \mathcal{P}(\mathbb{A} \times \mathbb{S}) \times \mathbb{Z} \rightarrow \mathbb{R}_{+}$such that

(i) Given the pricing functions $r$ and $w$, the policy function $f$ solves the following problem $v(a, s, z, \lambda)=\sup _{a^{\prime} \in \Gamma(a, s, z, \lambda)} u\left((1+r(\lambda, z)) a+w(\lambda, z) s-a^{\prime}\right)+\beta \int_{\mathbb{S} \times \mathbb{Z}} v^{\prime}\left(a^{\prime}, s^{\prime}, z^{\prime}, \lambda^{\prime}\right) Q\left(d s^{\prime}, d z^{\prime}, s, z\right)$, where $v \in \mathbb{C}(\mathbb{A} \times \mathbb{S} \times \mathbb{Z} \times \mathcal{P}(\mathbb{A} \times \mathbb{S}))$ and

$$
v^{\prime}(\cdot)=T^{v}(z, \lambda, v)(\cdot) \in \mathbb{C}(\mathbb{A} \times \mathbb{S} \times \mathbb{Z} \times \mathcal{P}(\mathbb{A} \times \mathbb{S})) \text { and } \lambda^{\prime}=G\left(z, \lambda, v, z^{\prime}\right) .
$$

(ii) The firm maximizes profits so that $r$ and $w$ satisfy (14)-(15).

(iii) The sequence of aggregate distributions induced by $G$ is such that labor markets clear: $\int_{\mathbb{A} \times \mathbb{S}} s \lambda_{t}(d a, d s)=N\left(z^{t}\right), \forall z^{t} \in \mathbb{Z}^{t}$, where $\lambda_{t+1}=G\left(z_{t}, \lambda_{t}, v_{t}, z_{t+1}\right)$ and $\lambda_{0}$ is given.

(iv) The law of motion for aggregate distributions $G$ is generated by the individual optimal policy $f$, i.e., for all $A \times B \in \mathcal{B}(\mathbb{A}) \times \mathcal{B}(\mathbb{S})$,

$$
G\left(z, \lambda, v, z^{\prime}\right)(A \times B)=\int_{\mathbb{A} \times \mathbb{S}} \mathbf{1}_{A}(f(a, s, z, \lambda, v)) Q\left(B, z^{\prime}, s, z\right) \lambda(d a, d s) .
$$


Remark 4.1 If individual shocks and aggregate shocks are independent, then $Q\left(B, z^{\prime}, s, z\right)$ does not depend on $z^{\prime}$ so that $G$ does not depend on $z^{\prime}$. In this case, $\lambda^{\prime}=G(z, \lambda, v)$. Note that requirement (iv) embodies rational expectations. It is justified by the analysis in Section 3.2 and Lemmas 2 and 4.

The following theorem shows that given an initial state, a recursive equilibrium generates a sequential competitive equilibrium.

Theorem 2. Let Assumptions 1-8 hold. Given the initial state $\left(\left(a_{0}^{i}, s_{0}^{i}\right)_{i \in I}, z_{0}, \lambda_{0}, v_{0}\right)$, a recursive equilibrium $\left(\left(f, T^{v}, G\right), r, w\right)$ generates a sequential competitive equilibrium $\left(\left(\left(a_{t+1}^{i}, c_{t}^{i}\right)_{t \geq 0}\right)_{i \in I}\right.$, $\left.\left(r_{t}, w_{t}\right)_{t \geq 0}\right)$ in which consumer $i$ 's expected discounted utilities are given by $v_{0}\left(a_{0}^{i}, s_{0}^{i}, z_{0}, \lambda_{0}\right)$.

The dynamics of the sequential competitive equilibrium $\left(\left(\left(a_{t+1}^{i}, c_{t}^{i}\right)_{t \geq 0}\right)_{i \in I},\left(r_{t}, w_{t}\right)_{t \geq 0}\right)$ is described as follows. Given the initial state $\left(\left(a_{0}^{i}, s_{0}^{i}\right)_{i \in I}, z_{0}, \lambda_{0}, v_{0}\right)$, the interest rate and the wage rate are given by $r_{0}=r\left(\lambda_{0}, z_{0}\right)$ and $w_{0}=w\left(\lambda_{0}, z_{0}\right)$, respectively. Consumer $i$ accumulates assets $a_{1}^{i}=f\left(a_{0}^{i}, s_{0}^{i}, z_{0}, \lambda_{0}, v_{0}\right)$ and consumes the remaining wealth $c_{0}^{i}=\left(1+r_{0}\right) a_{0}^{i}+w_{0} s_{0}^{i}-a_{1}^{i}$. At date 1 , when the realizations of individual shocks and aggregate shocks are $\left(s_{1}^{i}\right)_{i \in I}$ and $z_{1}$, the date 1 state $\left(\left(a_{1}^{i}, s_{1}^{i}\right)_{i \in I}, z_{1}, \lambda_{1}, v_{1}\right)$ is determined by the maps $\left(f, G, T^{v}\right)$. In particular, $\lambda_{1}=G\left(z_{0}, \lambda_{0}, v_{0}, z_{1}\right)$, $v_{1}=T^{v}\left(z_{0}, \lambda_{0}, v_{0}\right)$. Then the date 1 prices are given by $r_{1}=r\left(\lambda_{1}, z_{1}\right)$ and $w_{1}=w\left(\lambda_{1}, z_{1}\right)$. Under these prices, consumer $i$ accumulates assets $a_{2}^{i}=f\left(a_{1}^{i}, s_{1}^{i}, z_{1}, \lambda_{1}, v_{1}\right)$ and consumes the remaining wealth $c_{1}^{i}=\left(1+r_{1}\right) a_{1}^{i}+w_{1} s_{1}^{i}-a_{2}^{i}$. The state then moves to date 2 , and so on. Finally, the expected payoff to consumer $i$ in the equilibrium $\left(\left(\left(a_{t+1}^{i}, c_{t}^{i}\right)_{t \geq 0}\right)_{i \in I},\left(r_{t}, w_{t}\right)_{t \geq 0}\right)$ is given by $v_{0}\left(a_{0}^{i}, s_{0}^{i}, z_{0}, \lambda_{0}\right)$.

Does a recursive equilibrium defined earlier exist? Can any sequential competitive equilibrium be generated by such a recursive equilibrium? The following theorem answers these questions.

Theorem 3. Under Assumptions 1-8, there exists a recursive equilibrium. Moreover, for any competitive equilibrium $\left(\left(\left(a_{t+1}^{i}, c_{t}^{i}\right)_{t \geq 0}\right)_{i \in I},\left(r_{t}, w_{t}\right)_{t \geq 0}\right)$ with the sequence of aggregate distributions $\mu^{*}$, there exists a payoff equivalent competitive equilibrium that is generated by a recursive equilibrium.

This theorem implies that a recursive equilibrium exists. Moreover, any payoff implied by a sequential competitive equilibrium can be generated by a recursive equilibrium. Notice that the 
sequential competitive equilibrium generated by the recursive equilibrium may be different from the equilibrium $\left(\left(\left(a_{t+1}^{i}, c_{t}^{i}\right)_{t \geq 0}\right)_{i \in I},\left(r_{t}, w_{t}\right)_{t \geq 0}\right)$. But they imply the same expected discounted utilities.

The key to the proof of the theorem is to construct an equilibrium sequence of aggregate distributions $\bar{\mu}=\left(\lambda_{t}\right)_{t \geq 0}$ such that its law of motion satisfies (iv) in Definition 2. This is achieved by taking a measurable selection $\xi$ from the correspondence $\varphi$. Then $\lambda_{t+1}$ is obtained as the second component of $\xi\left(z_{t}, \lambda_{t}, v_{t}\right)$. The payoff $v_{t+1}\left(a_{t+1}, s_{t+1}, z_{t+1}, \lambda_{t+1}\right)$ is obtained as the continuation utility at date $t+1, V\left(a_{t+1}, s_{t+1}, z_{t+1}, \xi\left(z_{t}, \lambda_{t}, v_{t}\right)\right)$, implied by the equilibrium sequence of aggregate distributions $\xi\left(z_{t}, \lambda_{t}, v_{t}\right)$ when the economy starts at date $t$. This reflects rational expectations formed at the previous date. Moreover, $v_{t+1}$ serves as a device to select the 'continuation' equilibrium $\xi\left(z_{t+1}, \lambda_{t+1}, v_{t+1}\right)$ when the economy starts at date $t+1$. Finally, since the dynamics of the constructed equilibrium is stationary, the maps $\left(f, T^{v}, G\right)$ can be constructed so that a recursive equilibrium is obtained.

Turn to another recursive characterization proposed by Krusell and Smith [24], which assumes that the aggregate distribution does constitute a sufficient endogenous (aggregate) state.

Definition 3. A KS-recursive (competitive) equilibrium $((v, h, H),(r, w))$ consists of a value function $v: \mathbb{A} \times \mathbb{S} \times \mathbb{Z} \times \mathcal{P}(\mathbb{A} \times \mathbb{S}) \rightarrow \mathbb{R}$, a measurable policy function $h: \mathbb{A} \times \mathbb{S} \times \mathbb{Z} \times \mathcal{P}(\mathbb{A} \times \mathbb{S}) \rightarrow \mathbb{A}$, a measurable map $H: \mathcal{P}(\mathbb{A} \times \mathbb{S}) \times \mathbb{Z}^{2} \rightarrow \mathcal{P}(\mathbb{A} \times \mathbb{S})$, and measurable pricing functions $r: \mathcal{P}(\mathbb{A} \times \mathbb{S})$ $\times \mathbb{Z} \rightarrow \mathbb{R}$ and $w: \mathcal{P}(\mathbb{A} \times \mathbb{S}) \times \mathbb{Z} \rightarrow \mathbb{R}_{+}$such that

(i) Given the function $H$ and the pricing functions $r$ and $w, v$ and $h$ solve the problem: $v(a, s, z, \lambda)=\sup _{a^{\prime} \in \Gamma(a, s, z, \lambda)} u\left((1+r(\lambda, z)) a+w(\lambda, z) s-a^{\prime}\right)+\beta \int_{\mathbb{S} \times \mathbb{Z}} v\left(a^{\prime}, s^{\prime}, z^{\prime}, \lambda^{\prime}\right) Q\left(d s^{\prime}, d z^{\prime}, s, z\right)$, subject to $\lambda^{\prime}=H\left(\lambda, z, z^{\prime}\right)$.

(ii) The firm maximizes profits so that $r$ and $w$ satisfy (14)-(15).

(iii) The sequence of aggregate distributions induced by $H$ is such that labor markets clear: $\int_{\mathbb{A} \times \mathbb{S}} s \lambda_{t}(d a, d s)=N\left(z^{t}\right), \forall z^{t} \in \mathbb{Z}^{t}$, where $\lambda_{t+1}=H\left(\lambda_{t}, z_{t}, z_{t+1}\right)$ and $\lambda_{0}$ is given.

(iv) The law of motion for aggregate distributions $H$ is generated by the individual optimal 
policy h, i.e., for all $A \times B \in \mathcal{B}(\mathbb{A}) \times \mathcal{B}(\mathbb{S})$,

$$
H\left(\lambda, z, z^{\prime}\right)(A \times B)=\int_{\mathbb{A} \times \mathbb{S}} \mathbf{1}_{A}(h(a, s, z, \lambda)) Q\left(B, z^{\prime}, s, z\right) \lambda(d a, d s) \cdot{ }^{11}
$$

It is straightforward to show that a KS-recursive equilibrium generates a sequential competitive equilibrium. Does a KS-recursive equilibrium exist? For a related finitely many agents economy, Kubler and Schmedders [25, Theorem 2] establish the existence of such a recursive equilibrium under the strong condition that the competitive equilibrium is globally unique for all possible initial values. Although one can state a similar result for the economies studied here, this strong condition cannot be checked from primitives. It is an open question whether a KS-recursive equilibrium exists without this condition.

\section{CONCLUSION}

In this paper, I describe the Bewley-style model with aggregate shocks in terms of sequences of aggregate distributions. Using this formulation, I resolve two central open questions. Specifically, I establish the existence of a sequential competitive equilibrium and provide a recursive characterization. There are still some open questions remaining. For example, how should one design efficient numerical methods to solve Bewley models with aggregate shocks? Does there exist a recursive equilibrium defined in Krusell and Smith [24]? I leave these questions for future research.

\footnotetext{
${ }^{11}$ This condition can be justified by a similar analysis to that in section 3.2 .
} 


\section{A Appendix}

\section{Proof of Lemma 1:}

Define an operator $T$ on $\mathbb{V}^{\infty}$ as follows. For $v \in \mathbb{V}^{\infty}$, let $t^{t h}$ component of $T v\left(a_{t}, s^{t}, z^{t}, \mu\right)$ be the expression

$$
\begin{aligned}
(T v)_{t}\left(a_{t}, s^{t}, z^{t}, \mu\right)= & \max _{a_{t+1} \in \Gamma\left(a_{t}, s_{t}, z_{t}, \lambda_{t}\left(z^{t}\right)\right)} u\left(\left(1+r_{t}\left(\lambda_{t}\left(z^{t}\right), z_{t}\right)\right) a_{t}+w_{t}\left(\lambda_{t}\left(z^{t}\right), z_{t}\right) s_{t}-a_{t+1}\right) \\
& +\beta \int_{\mathbb{S} \times \mathbb{Z}} v_{t+1}\left(a_{t+1}, s^{t+1}, z^{t+1}, \mu\right) Q_{t+1}\left(d s_{t+1}, d z_{t+1}, s^{t}, z^{t}\right),
\end{aligned}
$$

I first show that $T v \in \mathbb{V}^{\infty}$. It is immediate that each $(T v)_{t}$ is bounded. To show continuity of $(T v)_{t}$, I apply the Maximum Theorem. Consider a sequence $\left(a_{t+1}, a_{t}, s^{t}, z^{t}, \mu\right)^{n} \rightarrow$ $\left(a_{t+1}, a_{t}, s^{t}, z^{t}, \mu\right), n=1,2, \ldots$ Since $\mathbb{Z}$ is countable, $\left(z^{t}\right)^{n}=z^{t}$ for all $n$ large enough. By (9)-(10) and the definition of weak convergence, $r_{t}\left(\lambda_{t}^{n}\left(\left(z^{t}\right)^{n}\right),\left(z_{t}\right)^{n}\right) \rightarrow r_{t}\left(\lambda_{t}\left(z^{t}\right), z_{t}\right), w_{t}\left(\lambda_{t}^{n}\left(\left(z^{t}\right)^{n}\right),\left(z_{t}\right)^{n}\right) \rightarrow$ $w_{t}\left(\lambda_{t}\left(z^{t}\right), z_{t}\right)$. Thus, $\Gamma$ is a continuous correspondence. Moreover, the first term on the right-hand side of $(22)$ is continuous in $\left(a_{t+1}, a_{t}, s^{t}, z^{t}, \mu\right)$ since $u$ is continuous.

Turn to continuity of the second term. For $n$ sufficiently large,

$$
\begin{aligned}
& \int_{\mathbb{S} \times \mathbb{Z}} v_{t+1}\left(\left(a_{t+1}\right)^{n},\left(s^{t}\right)^{n}, s_{t+1},\left(z^{t}\right)^{n}, z_{t+1}, \mu^{n}\right) Q_{t+1}\left(d s_{t+1}, d z_{t+1},\left(s^{t}\right)^{n},\left(z^{t}\right)^{n}\right) \\
= & \int_{\mathbb{S} \times \mathbb{Z}} v_{t+1}\left(\left(a_{t+1}\right)^{n},\left(s^{t}\right)^{n}, s_{t+1}, z^{t+1}, \mu^{n}\right) Q_{t+1}\left(d s_{t+1}, d z_{t+1},\left(s^{t}\right)^{n}, z^{t}\right) .
\end{aligned}
$$

Thus, it is sufficient to show that the following expression converges to zero:

$$
\begin{aligned}
& \mid \int_{\mathbb{S} \times \mathbb{Z}} v_{t+1}\left(\left(a_{t+1}\right)^{n},\left(s^{t}\right)^{n}, s_{t+1}, z^{t+1}, \mu^{n}\right) Q_{t+1}\left(d s_{t+1}, d z_{t+1},\left(s^{t}\right)^{n}, z^{t}\right) \\
& \quad-\int_{\mathbb{S} \times \mathbb{Z}} v_{t+1}\left(a_{t+1}, s^{t+1}, z^{t+1}, \mu\right) Q_{t+1}\left(d s_{t+1}, d z_{t+1}, s^{t}, z^{t}\right) \mid \\
& \leq \mid \int_{\mathbb{S} \times \mathbb{Z}} v_{t+1}\left(\left(a_{t+1}\right)^{n},\left(s^{t}\right)^{n}, s_{t+1}, z^{t+1}, \mu^{n}\right) Q_{t+1}\left(d s_{t+1}, d z_{t+1},\left(s^{t}\right)^{n}, z^{t}\right) \\
& \quad-\int_{\mathbb{S} \times \mathbb{Z}} v_{t+1}\left(a_{t+1}, s^{t+1}, z^{t+1}, \mu\right) Q_{t+1}\left(d s_{t+1}, d z_{t+1},\left(s^{t}\right)^{n}, z^{t}\right) \mid \\
& \quad+\mid \int_{\mathbb{S} \times \mathbb{Z}} v_{t+1}\left(a_{t+1}, s^{t+1}, z^{t+1}, \mu\right) Q_{t+1}\left(d s_{t+1}, d z_{t+1},\left(s^{t}\right)^{n}, z^{t}\right) \\
& \quad-\int_{\mathbb{S} \times \mathbb{Z}} v_{t+1}\left(a_{t+1}, s^{t+1}, z^{t+1}, \mu\right) Q_{t+1}\left(d s_{t+1}, d z_{t+1}, s^{t}, z^{t}\right) \mid .
\end{aligned}
$$

Since $\left(\left(a_{t+1}\right)^{n},\left(s^{t}\right)^{n}, s_{t+1}, z^{t+1}, \mu^{n}\right) \rightarrow\left(a_{t+1}, s^{t+1}, z^{t+1}, \mu\right)$, there is a compact set $D \subset \mathbb{A} \times \mathbb{S}^{t+1} \times$ $\mathbb{Z}^{t+1} \times \mathcal{P}_{\infty}(\mathbb{A} \times \mathbb{S})$ such that $\left(\left(a_{t+1}\right)^{n},\left(s^{t}\right)^{n}, s_{t+1}, z^{t+1}, \mu^{n}\right) \in D$ for all $n$ large enough, and 
$\left(a_{t+1}, s^{t+1}, z^{t+1}, \mu\right) \in D$. Since $v_{t+1}$ is continuous, it is uniformly continuous on $D$. Thus, for every $\varepsilon>0$, there exists $N>1$ such that for all $n>N, s_{t+1} \in \mathbb{S}$, and $z^{t+1} \in \mathbb{Z}^{t+1}$,

$$
\left|v_{t+1}\left(\left(a_{t+1}\right)^{n},\left(s^{t}\right)^{n}, s_{t+1}, z^{t+1}, \mu^{n}\right)-v_{t+1}\left(a_{t+1}, s^{t+1}, z^{t+1}, \mu\right)\right|<\varepsilon .
$$

This implies that the first absolute value in (23) vanishes as $n \rightarrow \infty$. The second absolute value also vanishes by the Feller property.

Next, $T$ is a contraction by a straightforward application of the Blackwell Theorem adapted to the infinite dimensional space $\mathbb{V}^{\infty}$ (see [12, Lamma A.1]). Finally, applying the Contraction Mapping Theorem and the Maximum Theorem yields the desired results.

\section{Proof of Lemma 2:}

See the main text.

\section{Proof of Lemma 3:}

I first show $\widehat{\mathcal{P}}\left(\mathbb{A} \times \mathbb{S}^{t}\right)$ is compact. Then $\widehat{\mathcal{P}}_{\infty}\left(\mathbb{A} \times \mathbb{S}^{t}\right)$ is also compact under the product topology. For any $\lambda \in \widehat{\mathcal{P}}\left(\mathbb{A} \times \mathbb{S}^{t}\right)$ and $a^{0}>0$,

$$
\widehat{K} \geq \int_{\mathbb{A} \times \mathbb{S}^{t}} a \lambda\left(d a, d s^{t}\right) \geq \int_{\left[a^{0}, \infty\right] \times \mathbb{S}^{t}} a \lambda\left(d a, d s^{t}\right) \geq a^{0} \lambda\left(\left[a^{0}, \infty\right) \times \mathbb{S}^{t}\right) .
$$

This implies that for any $\varepsilon>0$, there exists an $a^{0}$ large enough such that $\lambda\left(\left[a^{0}, \infty\right) \times \mathbb{S}^{t}\right)<\varepsilon$. Thus, $\widehat{\mathcal{P}}\left(\mathbb{A} \times \mathbb{S}^{t}\right)$ is tight and hence relatively compact (see [3, Theorem 14.22]). Furthermore, $\widehat{\mathcal{P}}\left(\mathbb{A} \times \mathbb{S}^{t}\right)$ is closed with respect to the weak convergence topology. It follows that $\widehat{\mathcal{P}}\left(\mathbb{A} \times \mathbb{S}^{t}\right)$ is compact.

\section{Proof of Theorem 1:}

I verify that the map $\psi: \widehat{\mathcal{P}}_{\infty}(\mathbb{A} \times \mathbb{S}) \rightarrow \mathcal{P}_{\infty}(\mathbb{A} \times \mathbb{S})$ defined in Section 3.3 satisfies the conditions of the Brouwer-Schauder-Tychonoff Fixed Point Theorem ([3, Corollary 16.52]). I first show that $\psi$ maps from $\widehat{\mathcal{P}}_{\infty}(\mathbb{A} \times \mathbb{S})$ into itself. Let $\mu=\left(\lambda_{0}, \lambda_{1}, \ldots\right) \in \widehat{\mathcal{P}}_{\infty}(\mathbb{A} \times \mathbb{S})$. Then $\psi(\mu)=\widetilde{\mu}=$ 
$\left(\widetilde{\lambda}_{0}, \widetilde{\lambda}_{1}, \ldots\right)$ is defined as in (12). It follows from (12) and Assumption 5 that

$$
\begin{aligned}
\int_{\mathbb{A} \times \mathbb{S}^{t}} a \widetilde{\lambda}_{t+1}\left(d a, d s^{t+1}\right) & =\int_{\mathbb{A} \times \mathbb{S}^{t}} g_{t+1}\left(a_{t}, s^{t}, z^{t}, \mu\right) \lambda_{t}\left(d a_{t}, d s^{t}\right) \\
& \leq \int_{\mathbb{A} \times \mathbb{S}^{t}}\left[\left(1+r_{t}\left(\lambda_{t}, z_{t}\right)\right) a_{t}+w_{t}\left(\lambda_{t}, z_{t}\right) s_{t}\right] \lambda_{t}\left(d a_{t}, d s^{t}\right) \\
& =\left(1+r_{t}\left(\lambda_{t}, z_{t}\right)\right) K_{t}+w_{t}\left(\lambda_{t}, z_{t}\right) N_{t} \\
& =(1-\delta) K_{t}+z_{t} F\left(K_{t}, N_{t}\right) \\
& \leq(1-\delta) \widehat{K}+\bar{z} F(\widehat{K}, \widehat{N})=\widehat{K} .
\end{aligned}
$$

Thus, $\psi(\mu) \in \widehat{\mathcal{P}}_{\infty}(\mathbb{A} \times \mathbb{S})$.

Finally, I show that $\psi$ is continuous. Fix a history of aggregate shocks $z^{\infty}$. Let the sequence of aggregate shocks $\mu^{n} \rightarrow \mu(n \rightarrow \infty), \mu^{n}, \mu \in \widehat{\mathcal{P}}_{\infty}(\mathbb{A} \times \mathbb{S})$. Let $\widetilde{\mu}=\psi(\mu), \widetilde{\mu}^{n}=\psi\left(\mu^{n}\right)$, and $\widetilde{\mu}^{n}=\left(\widetilde{\lambda}_{t}^{n}\right)_{t \geq 0}, n=1,2, \ldots$ By definition of $\psi, \widetilde{\lambda}_{0}^{n}=\lambda_{0}^{n} \rightarrow \lambda_{0}=\widetilde{\lambda}_{0}$. For any $t \geq 0$, it follows from (12) that for any bounded and continuous function $h: \mathbb{A} \times \mathbb{S}^{t+1} \rightarrow \mathbb{R}$, as $n \rightarrow \infty$,

$$
\begin{aligned}
& \int_{\mathbb{A} \times \mathbb{S}^{t+1}} h\left(a_{t}, s^{t+1}\right) \widetilde{\lambda}_{t+1}^{n}\left(d a_{t}, d s^{t+1}\right) \\
= & \int_{\mathbb{A} \times \mathbb{S}^{t}} \int_{\mathbb{S}} h\left(g_{t+1}\left(a_{t}, s^{t}, z^{t}, \mu^{n}\right), s^{t+1}\right) Q_{t+1}\left(d s_{t+1}, z_{t+1}, s^{t}, z^{t}\right) \lambda_{t}^{n}\left(d a_{t}, d s^{t}\right)
\end{aligned}
$$

converges to

$$
\begin{aligned}
& \int_{\mathbb{A} \times \mathbb{S}^{t}} \int_{\mathbb{S}} h\left(g_{t+1}\left(a_{t}, s^{t}, z^{t}, \mu\right), s^{t+1}\right) Q_{t+1}\left(d s_{t+1}, z_{t+1}, s^{t}, z^{t}\right) \lambda_{t}\left(d a_{t}, d s^{t}\right) \\
= & \int_{\mathbb{A} \times \mathbb{S}^{t+1}} h\left(a_{t}, s^{t+1}\right) \widetilde{\lambda}_{t+1}\left(d a_{t}, d s^{t+1}\right),
\end{aligned}
$$

where I have used the facts that $\lambda_{t}^{n}$ converges to $\lambda_{t}$ weakly and that $g_{t+1}$ is continuous in $a_{t}, s^{t}$, and $\mu^{n}$ by Lemma 1 . Thus, $\widetilde{\lambda}_{t+1}^{n}$ converges to $\lambda_{t+1}$ weakly for all $t \geq 0$. This implies that $\widetilde{\mu}^{n} \rightarrow \widetilde{\mu}$, and hence $\psi$ is continuous.

\section{Proof of Lemma 4:}

(i) Let $\mathbb{W}$ denote the set of uniformly bounded and continuous real-valued functions on $\mathbb{A} \times \mathbb{S} \times$ $\mathbb{Z} \times \mathcal{P}_{\infty}(\mathbb{A} \times \mathbb{S})$, where $\mathcal{P}_{\infty}(\mathbb{A} \times \mathbb{S})=\times_{t=0}^{\infty} \mathcal{P}(\mathbb{A} \times \mathbb{S})^{\mathbb{Z}^{t}}$. Let $\mathbb{W}^{\infty}$ denote the set of sequences $\bar{W}=(W, W, W, \ldots$.$) of such functions. Note that \mathbb{W}^{\infty}$ is a complete metric space if endowed with the norm

$$
\|\bar{W}\|=\sup _{(a, s, z, \mu)}|W(a, s, z, \mu)|
$$


Let the pricing functions $r: \mathcal{P}(\mathbb{A} \times \mathbb{S}) \times \mathbb{Z} \rightarrow \mathbb{R}$ and $w: \mathcal{P}(\mathbb{A} \times \mathbb{S}) \times \mathbb{Z} \rightarrow \mathbb{R}_{+}$be defined as in (14)-(15).

Next, let $\bar{W}=(W, W, \ldots) \in \mathbb{W}^{\infty}$. Given any sequence of aggregate distributions $\left(\lambda_{t}\right)_{t \geq 0}$, rewrite problem $(22)$ as

$$
\begin{aligned}
(T \bar{W})_{t}\left(a_{t}, s_{t}, z_{t},\left(\lambda_{\tau}\right)_{\tau \geq t}\right)= & \sup _{a_{t+1} \in \Gamma\left(a_{t}, s_{t}, z_{t}, \lambda_{t}\right)} u\left(\left(1+r\left(\lambda_{t}, z_{t}\right)\right) a_{t}+w\left(\lambda_{t}, z_{t}\right) s_{t}-a_{t+1}\right) \\
& +\beta \int_{\mathbb{S} \times \mathbb{Z}} W\left(a_{t+1}, s_{t+1}, z_{t+1},\left(\lambda_{\tau}\right)_{\tau \geq t+1}\right) Q\left(d s_{t+1}, d z_{t+1}, s_{t}, z_{t}\right),
\end{aligned}
$$

where I have applied Assumptions 7-8. Since the expression on the right side of the above equation is a time invariant function of $\left(a_{t}, s_{t}, z_{t},\left(\lambda_{\tau}\right)_{\tau \geq t}\right)$, the operator $T$ maps a sequence of time invariant function to another sequence of time invariant function. Thus, the fixed point of $T$ is a sequence of time invariant function, denoted by $(V, V, \ldots)$ where $V: \mathbb{A} \times \mathbb{S} \times \mathbb{Z} \times \mathcal{P}_{\infty}(\mathbb{A} \times \mathbb{S}) \rightarrow \mathbb{R}$ is continuous. The corresponding sequence of optimal policies is also time invariant, denoted by $(g, g, \ldots)$ where $g: \mathbb{A} \times \mathbb{S} \times \mathbb{Z} \times \mathcal{P}_{\infty}(\mathbb{A} \times \mathbb{S}) \rightarrow \mathbb{R}$. Moreover, $g$ is continuous by the Maximum Theorem.

Part (ii) follows from Lemma 2 and Assumptions 7-8.

\section{Proof of Lemma 5:}

Using a similar argument surrounding Lemma 3, one can restrict the range of the correspondence $\mathcal{E}$ to be a compact space. By Theorem $1, \mathcal{E}$ is closed-valued. Thus, to show that $\mathcal{E}$ is upper hemicontinuous, it suffices to show that $\mathcal{E}$ has a closed graph by the Closed Graph Theorem $[3$, Theorem 16.12].

Let $\left(z^{n}, \lambda^{n}\right)$ be a sequence converging to $(z, \lambda), n=1,2, \ldots$ Let $\left(\left(\lambda_{t}\right)_{t \geq 0}\right)^{n} \in \mathcal{E}\left(z^{n}, \lambda^{n}\right)\left(\lambda_{0}=\lambda\right)$ be a sequence of equilibrium sequences of aggregate distributions that converges to $\left(\lambda_{t}\right)_{t \geq 0}$. One needs to show that $\left(\lambda_{t}\right)_{t \geq 0} \in \mathcal{E}(z, \lambda)$. To this end, I apply Lemma 4. I first show $\left(\lambda_{t}\right)_{t \geq 0}$ satisfies (18). By the definition of weak convergence, for any bounded and continuous function $f$ on $\mathbb{A} \times \mathbb{S}$

$$
\int_{\mathbb{A} \times \mathbb{S}} f(a, s) \lambda_{1}^{n}\left(z^{1}\right)(d a, d s)
$$

converges to

$$
\int_{\mathbb{A} \times \mathbb{S}} f(a, s) \lambda_{1}\left(z^{1}\right)(d a, d s)
$$


as $n \rightarrow \infty$. Let $g$ be the function defined in Lemma 4 (i). Since $g$ is continuous, as $n \rightarrow \infty$,

$$
\int_{\mathbb{A} \times \mathbb{S}} \int_{\mathbb{S}} f\left(g\left(a_{0}, s_{0}, z^{n},\left(\lambda_{\tau}^{n}\right)_{\tau \geq 0}\right), s^{\prime}\right) Q\left(d s^{\prime}, z_{1}, s_{0}, z_{0}\right) \lambda_{0}^{n}\left(d a_{0}, d s_{0}\right)
$$

converges to

$$
\int_{\mathbb{A} \times \mathbb{S}} \int_{\mathbb{S}} f\left(g\left(a_{0}, s_{0}, z,\left(\lambda_{\tau}\right)_{\tau \geq 0}\right), s^{\prime}\right) Q\left(d s^{\prime}, z_{1}, s_{0}, z_{0}\right) \lambda_{0}\left(d a_{0}, d s_{0}\right) .
$$

Since $\left(\left(\lambda_{t}\right)_{t \geq 0}\right)^{n} \in \mathcal{E}\left(z^{n}, \lambda^{n}\right)$, it follows from Lemma 4 that

$$
\int_{\mathbb{A} \times \mathbb{S}} f(a, s) \lambda_{1}^{n}\left(z^{1}\right)(d a, d s)=\int_{\mathbb{A} \times \mathbb{S}} \int_{\mathbb{S}} f\left(g\left(a_{0}, s_{0}, z^{n},\left(\lambda_{\tau}^{n}\right)_{\tau \geq 0}\right), s^{\prime}\right) Q\left(d s^{\prime}, z_{1}, s_{0}, z_{0}\right) \lambda_{0}^{n}\left(d a_{0}, d s_{0}\right) .
$$

Taking limits yields

$$
\int_{\mathbb{A} \times \mathbb{S}} f(a, s) \lambda_{1}\left(z^{1}\right)(d a, d s)=\int_{\mathbb{A} \times \mathbb{S}} \int_{\mathbb{S}} f\left(g\left(a_{0}, s_{0}, z,\left(\lambda_{\tau}\right)_{\tau \geq 0}\right), s^{\prime}\right) Q\left(d s^{\prime}, z_{1}, s_{0}, z_{0}\right) \lambda_{0}\left(d a_{0}, d s_{0}\right) .
$$

Since this is true for any bounded and continuous function $f$, it follows that

$$
\lambda_{1}\left(z^{1}\right)(A \times B)=\int_{\mathbb{A} \times \mathbb{S}} \mathbf{1}_{A}\left(g\left(a_{0}, s_{0}, z,\left(\lambda_{\tau}\right)_{\tau \geq 0}\right) Q\left(B, z_{1}, s_{0}, z_{0}\right) \lambda_{0}\left(d a_{0}, d s_{0}\right),\right.
$$

for any $A \times B \in \mathcal{B}(\mathbb{A}) \times \mathcal{B}(\mathbb{S})$. Thus, Eq. (18) holds for $t=0$. Similarly, one can derive that for any $t \geq 1, \lambda_{t}$ satisfies (18).

Finally, since each $\left(\left(\lambda_{t}\right)_{t \geq 0}\right)^{n}$ satisfies (17), the limit $\left(\lambda_{t}\right)_{t \geq 0}$ also satisfies (17). Thus, by Lemma $4,\left(\lambda_{t}\right)_{t \geq 0}$ is an equilibrium sequence of aggregate distributions, i.e., $\left(\lambda_{t}\right)_{t \geq 0} \in \mathcal{E}(z, \lambda)$.

\section{Proof of Lemma 6:}

By a similar argument to that in Lemma 5, it suffices to show that $\varphi$ has a closed graph. This follows immediately from its definition and the fact that $V$ is continuous and $\mathcal{E}$ is upper hemicontinuous established in Lemma 5.

\section{Proof of Theorem 2:}

I show that the tuple $\left(\left(\left(a_{t+1}^{i}, c_{t}^{i}\right)_{t \geq 0}\right)_{i \in I},\left(r_{t}, w_{t}\right)_{t \geq 0}\right)$ described below the statement of Theorem 2 in the main text constitutes a sequential competitive equilibrium. First, it is clear that given prices $\left(r_{t}\right)$ and $\left(w_{t}\right)$, the firm maximizes profits. Second, I verify the market clearing condition. 
Integrating with respect to the measure $\phi$ yields:

$$
\begin{aligned}
C_{t}+K_{t+1} & =\int_{I} c_{t}^{i} \phi(d i)+\int_{I} a_{t+1}^{i} \phi(d i) \\
& =\left(1+r_{t}\right) \int_{I} a_{t}^{i} \phi(d i)+w_{t} \int_{I} s_{t}^{i} \phi(d i) \\
& =\left(1+r_{t}\right) K_{t}+w_{t} N_{t} \\
& =z_{t} F\left(K_{t}, N_{t}\right)+(1-\delta) K_{t}
\end{aligned}
$$

where the last equality follows from the construction of $r_{t}$ and $w_{t}$ and the homogeneity of $F$. Finally, given the constructed sequence of aggregate distributions $\left(\lambda_{t}\right)_{t \geq 0}$, by part (i) in Definition 2 and the principle of optimality, one can show that for any consumer $i,\left(a_{t+1}^{i}, c_{t}^{i}\right)_{t \geq 0}$ is optimal. ${ }^{12}$ Moreover, the implied expected discounted utilities are given by $v_{0}\left(a_{0}^{i}, s_{0}^{i}, z_{0}, \lambda_{0}\right)$.

\section{Proof of Theorem 3:}

I first construct a recursive equilibrium. I then show that for any sequential competitive equilibrium $\left(\left(\left(a_{t+1}^{i}, c_{t}^{i}\right)_{t \geq 0}\right)_{i \in I},\left(r_{t}, w_{t}\right)_{t \geq 0}\right)$ with implied sequence of aggregate distributions $\mu^{*}$, there exists another payoff equivalent sequential competitive equilibrium, that is generated by the recursive equilibrium.

Step 1. By Lemma 4, there exist continuous functions $V$ and $g$ solving the dynamic programming problem (16). By Theorem 1, a sequential competitive equilibrium exists. Thus the correspondence $\varphi$ defined in (19) is well defined. Since it is upper hemicontinuous by Lemma 6 , it follows from [18] that there exists a measurable selection $\xi$ from $\varphi$. I use $\xi$ to construct a recursive equilibrium with the expanded state space.

Define the pricing functions $(r, w)$ as in (14)-(15). Define the maps

$$
\begin{aligned}
f(a, s, z, \lambda, v) & =g(a, s, z, \xi(z, \lambda, v)), \\
G\left(z, \lambda, v, z^{\prime}\right)(A \times B) & =\int_{\mathbb{A} \times \mathbb{S}} \mathbf{1}_{A}\left(g(a, s, z, \xi(z, \lambda, v)) Q\left(B, z^{\prime}, s, z\right) \lambda(d a, d s),\right.
\end{aligned}
$$

where $A \times B \in \mathcal{B}(\mathbb{A}) \times \mathcal{B}(\mathbb{S})$. Since by definition $\xi(z, \lambda, v)$ is an equilibrium sequence of aggregate distributions, it must satisfy (18) in Lemma 4. Thus, by the above construction of $G\left(z, \lambda, v, z^{\prime}\right)$, $G\left(z, \lambda, v, z^{\prime}\right)$ is in fact the second component of the sequence of aggregate distributions $\xi(z, \lambda, v)$. Let $\lambda^{\prime}=G\left(z, \lambda, v, z^{\prime}\right)$. Thus, given the state $(z, \lambda, v)$ today and the aggregate shock $z^{\prime}$ tomorrow,

\footnotetext{
${ }^{12}$ See [33, Theorem 9.2]. Although the problem here is slightly different, one can use a similar repeated substitution method to prove the optimality.
} 
$\lambda^{\prime}$ is the equilibrium aggregate distribution tomorrow. Define

$$
T^{v}(z, \lambda, v)\left(a^{\prime}, s^{\prime}, z^{\prime}, \lambda^{\prime}\right)=V\left(a^{\prime}, s^{\prime}, z^{\prime}, \xi(z, \lambda, v)\right)
$$

I claim that $\left(\left(f, T^{v}, G\right),(r, w)\right)$ is a recursive equilibrium.

To verify this, one only needs to check part (i) in Definition 2 since part (ii)-(iv) are easily verified. To this end, consider the following dynamic programming problem

$$
\begin{aligned}
V(a, s, z, \xi(z, \lambda, v))= & \sup _{a^{\prime} \in \Gamma(a, s, z, \lambda)} u((1+r(\lambda, z)) a+w(\lambda, z) s-a) \\
& +\beta \int_{\mathbb{S} \times \mathbb{Z}} V\left(a^{\prime}, s^{\prime}, z^{\prime}, \xi(z, \lambda, v)\right) Q\left(d s^{\prime}, d z^{\prime}, s, z\right) .
\end{aligned}
$$

By construction and Lemma 4, the policy function $f(a, s, z, \lambda, v)=g(a, s, z, \xi(z, \lambda, v))$ solves the above problem. By the definition of $\xi$ and $\varphi$ in (19), and the above construction of $T^{v}$, problem (24) can be rewritten as

$$
\begin{aligned}
v(a, s, z, \lambda)= & \sup _{a^{\prime} \in \Gamma(a, s, z, \lambda)} u\left((1+r(\lambda, z)) a+w(\lambda, z) s-a^{\prime}\right) \\
& +\beta \int_{\mathbb{S} \times \mathbb{Z}} T^{v}(z, \lambda, v)\left(a^{\prime}, s^{\prime}, z^{\prime}, \lambda^{\prime}\right) Q\left(d s^{\prime}, d z^{\prime}, s, z\right) .
\end{aligned}
$$

This verifies part (i) in Definition 2.

Step 2. Consider the sequential competitive equilibrium $\left(\left(\left(a_{t+1}^{i}, c_{t}^{i}\right)_{t \geq 0}\right)_{i \in I},\left(r_{t}, w_{t}\right)_{t \geq 0}\right)$ with implied sequence of aggregate distributions $\mu^{*}$. Let $v_{0}\left(a_{0}^{i}, s_{0}^{i}, z_{0}, \lambda_{0}\right)=V\left(a_{0}^{i}, s_{0}^{i}, z_{0}, \mu^{*}\right)$ be the expected discounted utilities of consumer $i$. Then, one can use the argument after Theorem 2 in the main text to show that given the initial state $\left(\left(a_{0}^{i}, s_{0}^{i}\right)_{i \in I}, z_{0}, \lambda_{0}, v_{0}\right)$, the constructed recursive equilibrium generates a sequential competitive equilibrium, in which each consumer $i$ has expected discounted utilities $v_{0}\left(a_{0}^{i}, s_{0}^{i}, z_{0}, \lambda_{0}\right)=V\left(a_{0}^{i}, s_{0}^{i}, z_{0}, \mu^{*}\right)$. 


\section{References}

[1] D. Abreu, D. Pearce, and E. Stacchetti, Toward a theory of discounted repeated games with imperfect monitoring, Econometrica, 58 (1990) 1041-1063.

[2] S.R. Aiyagari, Uninsured idiosyncratic risk and aggregate saving, Quart. J. Econ. 109 (1994) 659-684.

[3] C.D. Aliprantis and K.C. Border, "Infinite Dimensional Analysis", 2nd Ed., Springer-Verlag Berlin, 1999.

[4] R.A. Becker and I. Zilcha, Stationary Ramsey equilibria under uncertainty, J. Econ. Theory, 75 (1997) 122-140.

[5] J. Bergin, A characterization of sequential equilibrium strategies in infinitely repeated incomplete information games, J. Econ. Theory, 47 (1989) 51-65.

[6] J. Bergin and D. Bernhardt, Anonymous sequential games with aggregate uncertainty, J. Math. Econ., 21 (1992) 543-562.

[7] J. Bergin and D. Bernhardt, Anonymous sequential games: existence and characterization of equilibria, Econ. Theory, 5 (1995) 461-489.

[8] T. Bewley, Stationary monetary equilibrium with a continuum of independently fluctuating consumers, in "Contributions to Mathematical Economics in Honor of Gerard Debreu" (W. Hildenbrand and A. Mas-Colell, Eds.), Amsterdam: North Holland, 1986.

[9] R.H. Clarida, International lending and borrowing in a stochastic stationary equilibrium, Inter. Econ. Rev. 31 (1990) 543-558.

[10] P. Dubey and L.S. Shapley, Noncooperative general exchange with a continuum of traders: two models, J. Math. Econ., 23 (1994) 253-293.

[11] D. Duffie, J. Geanakoplos, A. Mas-Colell, and A. McLennan, Stationary Markov equilibria, Econometrica, 62 (1994) 745-781.

[12] L.G. Epstein and T. Wang, Intertemporal asset pricing under Knightian uncertainty, Econometrica, 62 (1994) 283-322.

[13] M. Feldman and C. Gilles, An expository note on individual risk without aggregate uncertainty, J. Econ. Theory, 35 (1985) 26-32. 
[14] P.O. Gourinchas, Precautionary saving, life cycle, and macroeconomics, Working paper, Princeton University, 2000.

[15] S. Hart, W. Hildenbrand and E. Kohlberg, On equilibrium allocations as distributions on the commodity space, J. Math. Econ., 1 (1974) 159-167.

[16] J. Heaton and D. Lucas, The importance of investor heterogeneity and financial market imperfections for the behavior of asset prices, Carnegie-Rochester Conference Series on Public Policy, 42 (1995) 1-32.

[17] A.D. Hernandez and M.S. Santos, Competitive equilibria for infinite-horizon economies with incomplete markets, J. of Econ. Theory, 71 (1996), 102-130.

[18] W. Hildenbrand, "Core and Equilibria of a Large Economy", Princeton University Press, Princeton, NJ, 1974.

[19] M. Huggett, The risk-free rate in heterogeneous-agent incomplete-insurance economies, $J$. Econ. Dynam. and Control., 17 (1993) 953-969.

[20] K.L. Judd, The law of large numbers with a continuum of IID random variables, J. Econ. Theory, 35 (1985) 19-25.

[21] I. Karatzas, M. Shubik, and W. Sudderth, Construction of a stationary Markov equilibria in a strategic market game, Math. Oper. Res. 19 (1994) 975-1006.

[22] T. Krebs, Non-Existence of recursive equilibria on compact state spaces when markets are incomplete, J. of Econ. Theory, 115 (2004), 134-150.

[23] P. Krusell and A.A. Smith, Jr., Income and wealth heterogeneity, portfolio choice and equilibrium asset returns, Macroecon. dynam., 1 (1997) 387-422.

[24] P. Krusell and A.A. Smith, Jr., Income and wealth heterogeneity in the macroeconomy, J. Pol. Econ., 105 (1998) 867-896.

[25] F. Kubler and K. Schmedders, Recursive equilibria in economies with incomplete markets, Macroecon. dynam., 6 (2002) 284-306.

[26] F. Kubler and K. Schmedders, Stationary equilibria in asset-pricing models with incomplete markets and collateral, Econometrica, 71 (2003), 1767-1793. 
[27] D. Levine and W. Zame, Debt constraints and equilibrium in infinite horizon economies with incomplete markets, J. of Math. Econ., 26 (1996), 103-131.

[28] L. Ljunqvist and T. Sargent, "Recursive Macroeconomic Theory", 2000, MIT Press.

[29] M. Magill and M. Quinzii, Infinite horizon incomplete markets, Econometrica 62 (1994), 853-880.

[30] M. Magill and M. Quinzii, Incomplete markets over an infinite horizon: long-lived securities and speculative bubbles, J. of Math. Econ. 26 (1996) 133-170.

[31] J. Miao, Stationary equilibria of economies with a continuum of consumers, Working paper, Boston University, 2002.

[32] S.E. Spear and S. Srivastava, On repeated moral hazard with discounting, Rev. Econ. Studies 54 (1987) 599-617.

[33] N. Stokey and R.E. Lucas with E. Prescott, "Recursive Methods in Economic Dynamics", Harvard University Press, 1989.

[34] K. Storesletten, C. Telmer, A. Yaron, Asset pricing with idiosyncratic risks and overlapping generation, Working paper, Carnegie Mellon University, 2001.

[35] Y.N. Sun, A theory of hyperfinite processes: the complete removal of individual uncertainty via exact LLN, J. Math. Econ. 29 (1998), 419-503.

[36] J. Thomas and T. Worrall, Self-enforcing wage contracts, Rev. Econ. Studies, 55 (1988) $541-554$. 